\title{
Intergalactic Magnetic Fields from Quasar Outflows
}

\section{Citation}

Furlanetto, Steven R., and Abraham Loeb. 2001. “Intergalactic Magnetic Fields from Quasar Outflows." The Astrophysical Journal 556 (2): 619-34. https://doi.org/10.1086/321630.

\section{Permanent link}

http://nrs.harvard.edu/urn-3:HUL.InstRepos:41393295

\section{Terms of Use}

This article was downloaded from Harvard University's DASH repository, and is made available under the terms and conditions applicable to Other Posted Material, as set forth at http:// nrs.harvard.edu/urn-3:HUL.InstRepos:dash.current.terms-of-use\#LAA

\section{Share Your Story}

The Harvard community has made this article openly available.

Please share how this access benefits you. Submit a story.

Accessibility 
The Astrophysical Journal, 556:619-634, 2001 August 1

(C) 2001. The American Astronomical Society. All rights reserved. Printed in U.S.A.

\title{
INTERGALACTIC MAGNETIC FIELDS FROM QUASAR OUTFLOWS
}

\author{
SteVen R. Furlanetto and Abraham Loeb \\ Harvard-Smithsonian Center for Astrophysics,60 Garden Street, Cambridge, MA 02138; sfurlanetto@cfa.harvard.edu, aloeb@cfa.harvard.edu \\ Received 2001 February 5 ; accepted 2001 April 9
}

\begin{abstract}
Outflows from quasars inevitably pollute the intergalactic medium (IGM) with magnetic fields. The short-lived activity of a quasar leaves behind an expanding magnetized bubble in the IGM. We model the expansion of the remnant quasar bubbles and calculate their distribution as a function of size and magnetic field strength at different redshifts. We generically find that by a redshift $z \sim 3$, about $5 \%-20 \%$ of the IGM volume is filled by magnetic fields with an energy density $\gtrsim 10 \%$ of the mean thermal energy density of a photoionized IGM (at $\sim 10^{4} \mathrm{~K}$ ). As massive galaxies and X-ray clusters condense out of the magnetized IGM, the adiabatic compression of the magnetic field could result in the field strength observed in these systems without a need for further dynamo amplification. The intergalactic magnetic field could also provide a nonthermal contribution to the pressure of the photoionized gas that may account for the claimed discrepancy between the simulated and observed Doppler width distributions of the $\operatorname{Ly} \alpha$ forest.
\end{abstract}

Subject headings: cosmology: theory — intergalactic medium — magnetic fields - quasars: general

\section{INTRODUCTION}

The interstellar medium of galaxies is known to possess strong magnetic fields that are dynamically important and reach near equipartition with the total (turbulent plus thermal) kinetic energy (Zeldovich, Ruzmaikin, \& Sokoloff 1983; Beck et al. 1996). Substantial magnetic fields are also known to exist on larger cosmological scales, but their dynamical significance is uncertain. Faraday rotation measurements imply a lower limit of $1-100 \mu \mathrm{G}$ on the tangled magnetic field in the cores of X-ray clusters (Kim et al. 1990; Kim, Tribble, \& Kronberg 1991; Taylor \& Perley 1993; Ge \& Owen 1993; Dreher, Carilli, \& Perley 1987; Perley \& Taylor 1991), while the comparison between the synchrotron and inverse Compton emission by relativistic electrons in the outer $(\gtrsim 1 \mathrm{Mpc}$ ) envelopes of X-ray clusters indicates a lower limit of $\sim 0.1 \mu \mathrm{G}$ there (Fusco-Femiano et al. 1999; Rephaeli, Gruber, \& Blanco 1999; Kaastra et al. 1999; Kim et al. 1989). The observed field amplitude of $B \gtrsim 10^{-7} \mathrm{G}$ in collapsed environments with an overdensity factor of $\delta \sim 10^{3}$ translates to $B \gtrsim 10^{-9} \mathrm{G}$ at the mean density of the intergalactic medium (IGM), under the assumption that the field was adiabatically compressed $\left(B \propto \delta^{2 / 3}\right)$ as the gas collapsed. The corresponding intergalactic magnetic field (IGMF) should have an energy density of $B^{2} / 8 \pi \gtrsim 4$ $\times 10^{-20} \mathrm{ergs} \mathrm{cm}^{-3}$, which amounts to $\gtrsim 5 \%$ of the thermal energy density of the IGM at the mean cosmic density and the photoionization temperature of $T \sim 10^{4} \mathrm{~K}$, typical of uncollapsed regions. The existence of this nonthermal pressure component could have had a significant effect on the Ly $\alpha$ forest and on the fragmentation of the IGM into dwarf galaxies.

The origin of the observed magnetic field in galaxies and $\mathrm{X}$-ray clusters is still unresolved. The dynamo amplification process in galactic disks requires many dynamical times and cannot account for the tentative detection of magnetic fields in high-redshift galaxies (Oren \& Wolfe 1995). On the other hand, a cosmic origin in the early universe is problematic because the comoving scale of causally connected regions is small in the pre-recombination epoch (e.g., Quashnock, Loeb, \& Spergel 1989), and field amplification processes are typically weak subsequently (Harrison 1973; Gnedin,
Ferrara, \& Zweibel 2000). Kulsrud et al. (1997) proposed a scenario in which the fields are generated by the Biermann battery mechanism during structure formation and subsequently amplified through a turbulent energy cascade. However, as those authors acknowledge, it is not clear that the field will obtain the observed coherence on galactic scales after the cascade.

In this paper we explore the possibility that the observed galactic and intergalactic fields originate from energetic quasar outflows, an idea originally proposed by Rees \& Setti (1968) for intergalactic fields, by Hoyle (1969) for galactic fields, and examined more recently by MedinaTanco \& Enßlin (2000). These outflows can carry magnetic flux from the very compact accretion disk $\left(\lesssim 10^{15} \mathrm{~cm}\right)$ around a quasar black hole, where the growth time is very short, out to cosmological scales $\left(\sim 10^{24} \mathrm{~cm}\right)$, starting at high redshifts. The stretching of field lines by the outflows could also account for the coherent field structure that is observed in galactic disks (Daly \& Loeb 1990; Howard \& Kulsrud 1997; note that supernova-driven outflows cannot reach the same level of coherence) and explain the large magnetic flux that is observed in X-ray clusters (Colgate \& Li 2000). Quasar outflows are detected in two forms: (1) radio jets with strong magnetic fields in about a tenth of all quasars (Begelman, Blandford, \& Rees 1984) and (2) broad absorption line (BAL) outflows in a different tenth of the observed quasar population (possibly due to the covering fraction of the outflow; Weymann 1997) that are thought to carry magnetic fields as well (de Kool \& Begelman 1995). Both types of outflows may transport a substantial fraction of the accretion energy of the quasar into the surrounding medium (see, e.g., Begelman et al. 1984; Murray et al. 1995; Blandford \& Begelman 1999).

In addition to magnetizing the IGM, quasar outflows may have a variety of other consequences. Natarajan, Sigurdsson, \& Silk (1998) examined the possibility that such outflows can trigger the formation of baryon-rich dwarf galaxies, while Natarajan \& Sigurdsson (1999) argued that such outflows will produce potentially observable SunyaevZeldovich decrements in the cosmic microwave background. Mechanical outflows from quasars could also play 
a role in cosmological reionization. It is now known from observations of high-redshift quasars that the diffuse IGM was reionized at a redshift $z \gtrsim 6$ (Fan et al. 2000). Most scenarios for reionization assume that the energy source is radiation from quasars (Haiman \& Loeb 1998; Valageas \& Silk 1999) or stars (Haiman \& Loeb 1997; Chiu \& Ostriker 2000; Gnedin 2000), although some authors have also considered the effects of winds from starburst galaxies (Tegmark, Silk, \& Evrard 1993). It is important to examine the effects of quasar outflows because reionization via mechanical processes (either starburst or quasar outflows) would have been qualitatively different from photoionization. Simulations have shown that reionization in the latter case would have had the character of a phase transition (Gnedin 2000; Barkana \& Loeb 2001). This would not be true for reionization via collisional processes; in such a scenario the flux of ionizing particles at a given distance from the source is limited by the particle velocity rather than by the optical depth of the ambient medium. Thus, the ionizing agent cannot efficiently escape preionized regions, and we would expect reionization to occur over a much broader redshift interval than in the photoionization case.

Because of the short duration of the quasar activity, the outflow impulse leaves behind a magnetized high-pressure bubble that expands into the IGM. In $\S 2$ we model the expansion of such a bubble into the surrounding IGM and add up the cumulative effects of many quasar sources. In $\S 3$ we present numerical results from this model, and in $\S 4$ we examine their implications for the IGMF. Throughout the paper we assume a flat universe with a cosmological constant having $\Omega_{0}=0.3, \Omega_{\Lambda 0}=0.7, \Omega_{b} h^{2}=0.019, H_{0}=70$ $\mathrm{km} \mathrm{s}^{-1} \mathrm{Mpc}^{-1}$, and $\sigma_{8}=0.9$.

\section{METHOD}

\subsection{Bubble Expansion}

The expansion of an outflow from a quasar into the IGM depends on the kinetic energy output, the density distribution of ambient gas, and the gravitational potential well in which the quasar resides. In our models, we assume that a fixed fraction of all quasars expel magnetized winds that carry a fraction of the total quasar radiative luminosity. We embed each quasar in a galactic halo, adopting a simple prescription for the host properties that has been shown to be successful in describing the observed quasar luminosity function at $2 \lesssim z \lesssim 5$ (see the review by Barkana \& Loeb 2001 and references therein).

In the following subsections we describe our model for the expansion of the magnetized outflow around a quasar. We first identify the properties of a quasar host in $\S$ 2.1.1. We then consider the early expansion phase, during which the source is still active, in $\S 2.1 .2$. Once this active phase ends, the magnetized region relaxes into an approximately spherical shell. The overpressured interior forces the shell to expand, as described in $\S 2.1 .3$. Finally, in $\S 2.1 .4$ we show how the expansion depends on the input parameters and assumptions.

\subsubsection{Halo and Environment}

By now, it is widely accepted that quasars are active stages in the growth of the supermassive black holes at the centers of galaxies. In modeling the quasar host properties we adopt the simplest model of Haiman \& Loeb (1998), whereby each black hole amounts to a fixed fraction of the halo mass, $\epsilon_{h}$, and has a universal active lifetime, $\tau_{q}$, during which it shines at the Eddington limit with a universal spectrum. The two free parameters of this model can be chosen so as to reproduce the observed quasar luminosity function at moderate redshifts. The black hole mass may be written as

$$
M_{\mathrm{BH}}=\frac{1}{\epsilon_{\mathrm{bol}}}\left(\frac{L_{B}}{1.4 \times 10^{38} \mathrm{ergs} \mathrm{s}^{-1}}\right) M_{\odot},
$$

where $L_{B}$ is the rest-frame $B$-band luminosity of the quasar, $M_{\mathrm{BH}}$ is the mass of the central black hole, and $\epsilon_{\mathrm{bol}}$ is the fraction of the Eddington luminosity radiated in the $B$ band. The median quasar spectrum of Elvis et al. (1994) gives $\epsilon_{\mathrm{bol}}=0.093$, assuming that the quasar radiates at the Eddington limit.

Under the assumption that the central black hole of a galaxy contains a fixed fraction of its total baryonic mass (Magorrian et al. 1998; Gebhardt et al. 2000), we express the black hole mass as a fixed fraction of the total galaxy mass $M_{\mathrm{BH}}=\epsilon_{h} M_{h}$, where $\epsilon_{h} \approx\left(M_{\mathrm{BH}} / M_{\text {bulge }}\right)\left(\Omega_{b} / \Omega_{0}\right) \approx 4 \times 10^{-4}$, assuming the most recent value of $M_{\mathrm{BH}} / M_{\text {bulge }} \approx(2-3)$ $\times 10^{-3}$ (Gebhardt et al. 2000).

We assume that each quasar begins its active phase at the formation redshift of its host halo, $z_{0}$. Numerical simulations have shown that relaxed cold dark matter halos have a universal density profile within their virial radii over a broad range of masses (Navarro, Frenk, \& White 1997, hereafter NFW). The dark matter density profile of the host, $\rho_{d}(r)$, is

$$
\rho_{d}(r)=\rho_{c 0}\left(1+z_{0}\right)^{3} \frac{\Omega_{0}}{\Omega\left(z_{0}\right)} \frac{\delta_{c}}{\left(c r / R_{v}\right)\left(1+c r / R_{v}\right)^{2}},
$$

where $\rho_{c 0}$ is the critical density today, $\Omega\left(z_{0}\right)$ is the matter density parameter at redshift $z_{0}$, and $\delta_{c}$ is the characteristic density of the halo, determined by its mass and formation redshift (NFW). The concentration parameter $c$ is a function only of the chosen cosmology and of $\delta_{c}$,

$$
\delta_{c}=\frac{\Delta_{c}}{3} \frac{c^{3}}{\ln (1+c)-c /(1+c)} .
$$

Here $\Delta_{c}$ is the cosmology-dependent characteristic virial overdensity (Bryan \& Norman 1998). The term $R_{v}$ is the virial radius of the halo (Barkana \& Loeb 2001):

$$
\begin{aligned}
R_{v}= & 3.51\left(\frac{M_{h}}{10^{10} h^{-1} M_{\odot}}\right)^{1 / 3}\left[\frac{\Omega_{0}}{\Omega\left(z_{0}\right)} \frac{\Delta_{c}}{200}\right]^{-1 / 3} \\
& \times\left(\frac{1+z_{0}}{10}\right)^{-1} h^{-1} \mathrm{kpc} .
\end{aligned}
$$

The collisionless dark matter interacts only gravitationally with the quasar outflow. The baryons, on the other hand, interact directly with the outflow because the outflow must either sweep the gas aside (during a jet phase) or sweep it into a shell (if the outflow is spherical). X-ray observations exclude the existence of extended X-ray halos or cooling flows in most spiral galaxies in the local universe (Fabbiano 1989). Hence, we assume that all the gas within the virial radius of the halo cools onto the central core (disk or bulge) of the galaxy (see $\S 2.1 .4$ for a discussion of the importance of this assumption). 
Gas infall outside the halo results in a velocity field $v_{p}(r)$ directed toward the halo. There is no simple form for this field at low redshifts in an $\Omega_{\Lambda} \neq 0$ universe, so we make a crude approximation to the self-similar solution for a flat $\Omega_{0}=1$ universe given by Bertschinger (1985):

$$
v_{p}(r)= \begin{cases}0 & r<R_{v}, \\ \frac{\sigma}{3}\left(\frac{r}{R_{v}}-4\right) & R_{v}<r<4 R_{v}, \\ \frac{3 H}{2}\left(r-4 R_{v}\right) & 4 R_{v}<r<12 R_{v}, \\ H r & 12 R_{v}<r .\end{cases}
$$

Here $\sigma$ is the virial velocity dispersion of the halo (Barkana \& Loeb 2001):

$\sigma=223\left(\frac{R_{v}}{h^{-1} \mathrm{kpc}}\right)\left[\frac{\Omega_{0}}{\Omega\left(z_{0}\right)} \frac{\Delta_{c}}{200}\right]^{1 / 2}\left(\frac{1+z_{0}}{10}\right)^{3 / 2} \mathrm{~km} \mathrm{~s}^{-1}$.

Inside the accretion shock at the virial radius, the gas is assumed to be either cold or in hydrostatic equilibrium. (In our standard scenario, all of the gas inside the virial radius has cooled onto the galaxy, so the second possibility comes into play only when we examine the dependence of the model on our assumption of cooling in $\S 2.1 .4$.) Just outside of this radius gas falls onto the halo, while farther away the gas recedes with the Hubble flow. The precise form of the infall velocity field has only a small effect on the final results $(\$ 5 \%)$. The self-similar solution also determines the density distribution of the dark matter and gas through which the outflow must travel (Bertschinger 1985). Outside of the accretion shock both the dark matter and gaseous components follow the profile $\rho \propto r^{-2.25}$ for $\Omega_{b} \ll \Omega_{0}$. There are two important simplifications inherent to our treatment of this infall region. First, the self-similar solution applies to an $\Omega_{0}=1$ universe, while we use an $\Omega_{\Lambda} \neq 0$ cosmology. However, because quasars are most common at high redshifts where the effects of the cosmological constant are small, this introduces only small changes in our results. Second, we ignore the slow growth of the infall region as the halo accretes more mass. This is justified because those outflows that escape the gravitational potential of their host traverse the infall region quickly, before that region has evolved significantly.

We find in our calculations that the detailed density structure of the halo infall region has little effect on the results. Although the density solutions of Bertschinger (1985) and NFW differ substantially within the virial radius, the accumulated mass in the expanding shell is dominated by swept-up mass at large radii, at which the density is close to the cosmological mean in both cases. For computational convenience, we assume that the dark matter continues to follow the NFW profile outside of $R_{v}$ until $\rho_{d}$ falls below the mean cosmological density at $z_{0}$. Outside of this radius, we assume that the density field follows the (time-dependent) mean cosmological density. The simple prescription that the gas simply follows the dark matter, with a density $\rho_{g}=$ $\left(\Omega_{b} / \Omega_{0}\right) \rho_{d}$, results in only a minor loss of accuracy compared to the calculation with the full density profile of Bertschinger (1985).

We have now associated each quasar of rest-frame $B$-band luminosity $L_{B}$ with a host halo. Next, we assign a kinetic (or mechanical) luminosity $L_{K}=\epsilon_{K} L_{B}$ to each quasar. Recent evidence suggests that the kinetic and radiation luminosities are comparable for radio jets. Willott et al. (1999) have examined the correlation between narrow emission line luminosity (which is excited by radiation from the central engine and thus is related to the bolometric luminosity $L_{\mathrm{bol}}$ ) and radio luminosity (assumed to correlate with the jet power) in radio galaxies, over three decades in radio power. They found that their theoretical model, in which $L_{K} \propto L_{\text {bol }}$ and $0.05 \lesssim L_{K} / L_{\text {bol }} \lesssim 1.0$, was consistent with the available data. Because $L_{\mathrm{bol}} \approx 10 L_{B}$ in the restframe median quasar spectrum of Elvis et al. (1994), we conservatively adopt $\epsilon_{K}=1.0$ for radio-loud quasars (RLQs).

In BAL quasars, the relation between radiative and kinetic power is even more uncertain. Given the column density of absorbing gas $N_{\mathrm{H}}$, the mean outflow velocity $v_{\mathrm{BAL}}$, the radius of the absorbing system $R_{\mathrm{BAL}}$, and its covering fraction of the central source $f_{c}$, the kinetic luminosity of the system is

$$
L_{K} \approx 2 \pi f_{c} N_{\mathrm{H}} m_{p} v_{\mathrm{BAL}}^{3} R_{\mathrm{BAL}},
$$

where $m_{p}$ is the proton mass. Of these quantities, the best known is $v_{\mathrm{BAL}} \lesssim 0.1 c$, which can be directly inferred from the absorption lines. Unified models suggest that $f_{c} \sim 0.1$, although this value is highly uncertain and could vary among individual sources (Weymann 1997). The radius $R_{\mathrm{BAL}}$ can be constrained based on the photoionizing flux of the quasar, and estimates for individual sources cover the range $R_{\mathrm{BAL}} \sim 1-500 \mathrm{pc}$ (Krolik 1999; Turnshek et al. 1994). The value of $N_{\mathrm{H}}$ can be constrained with X-ray absorption data. ASCA measurements have recently indicated much higher column densities of absorbing gas than previously suspected, $N_{\mathrm{H}} \approx 10^{22}-10^{23} \mathrm{~cm}^{-2}$ (Gallagher et al. 1999). Given that the inferred column density of this gas is correlated with that of the UV-absorbing gas (Gallagher et al. 2001), our estimates for individual quasars range from $\epsilon_{K} \sim$ 0.01 to 10 . Again, $\epsilon_{K}=1.0$ appears to be a reasonable choice.

\subsubsection{Early Expansion and Isotropization}

Expansion during the initial phase, while the quasar is active, depends strongly on the geometry of the outflow. For an RLQ, the outflow is tightly collimated, as observed in nearby galaxies. The geometry of a BAL quasar outflow is unknown. For concreteness, we begin with a discussion of outflow in jets.

During the active lifetime of an RLQ, we assume that the outflow is collimated in twin jets. The material in radio jets is known to be highly relativistic near the central source and at least moderately relativistic away from the nucleus (Begelman et al. 1984; Tingay et al. 2001; Biretta, Zhou, \& Owen 1995). The momentum flux in each jet is therefore $L_{K} /(2 c)$. As the jet strikes the ambient medium, it forms a contact discontinuity between forward and reverse shocks (Begelman et al. 1984). The expansion rate of the contact discontinuity can be found by balancing the outward momentum flux of the particles in the jet with the external ram pressure of the medium:

$$
\frac{L_{K}}{2 A_{s} c}=\rho_{g}\left(v_{s}-v_{p}\right)^{2},
$$

where $A_{s}$ is the total area of the interface (generally larger than the instantaneous area of the jet) and $v_{s}$ is the velocity 
of the discontinuity. We assume that the shock front has a constant half-opening angle $\theta=5^{\circ}$ relative to the central source (Bridle \& Perley 1984); this could correspond to either a shock front with a constant transverse expansion rate or a precessing, narrow jet. Note that observations show that $v_{s} \ll c$ under the conditions of interest here (Krolik 1999). This may also be written as

$$
\begin{aligned}
\frac{d R}{d t}-v_{p}= & 5.5 \times 10^{-3}\left(\frac{5^{\circ}}{\theta}\right)\left(\frac{L_{K}}{10^{46} \mathrm{ergs} \mathrm{s}^{-1}}\right)^{1 / 2} \\
& \times\left[\frac{\rho_{g}(R)}{10^{-29} \mathrm{~g} \mathrm{~cm}^{-3}}\right]^{-1 / 2}\left(\frac{R}{\mathrm{kpc}}\right)^{-1}\left(\frac{\mathrm{kpc}}{\mathrm{yr}}\right),
\end{aligned}
$$

where $R$ is the length of the jet (measured from the center of the host galaxy).

Once the quasar becomes dormant, we assume that the jets rapidly balloon outward to form a sphere with a radius equal to the final length of the jet. We then assume that the total mechanical outflow energy, $E_{K}=L_{K} \tau_{q}$, goes into the thermal energy of the bubble. Pressure gradients within this region will rapidly force most of the gas into a shell at the edge. The energy that remains inside the shell produces a hot, overpressured interior. During the formation of the thin shell, some of the thermal energy of the interior is lost to ionization processes and radiation. In the case of supernova remnants, $\sim 25 \%$ of the explosion energy remains in the thermal energy of the interior after the transition to the shell phase, while another $\sim 25 \%$ is contained in the kinetic energy of the shell (Cox 1972; Falle 1981). In our scenario, the remnants are considerably more rarefied, and so a much smaller fraction of the energy would be radiated away during the formation of the shell. (Note that in a cosmological context, even adiabatic blast waves quickly form thin shells; Ostriker \& McKee 1988.) The uncertainty about the energy loss during this process can be absorbed into the much larger uncertainty in $\epsilon_{K}$ (the significance of which will be discussed in $\S 2.1 .4$ ).

The assumption of spherically symmetric expansion may not be valid for all quasar environments. Because halos tend to form at the intersection of sheets and filaments, the density distribution around the halos will be inhomogeneous. The shells will therefore expand along the path of least resistance and preferentially in directions away from these filaments (Martel \& Shapiro 2001). This is in fact an important effect; taken literally, our model would suppress infall in all but the largest halos as the shell sweeps away the infalling matter. In reality, however, infall primarily occurs in channels along filaments and sheets. In these directions, the expansion of the shell will most likely be halted quickly and infall will continue. Meanwhile, in directions away from the filaments, the shell will continue to expand. Although this process will certainly affect the geometry of the resulting magnetic field, it may not strongly affect the overall filling factor given by our model. Including such geometric effects will, in any case, require the use of numerical simulations.

We also assume that the quasar expels magnetic energy during its active phase. Unfortunately, the behavior of the magnetic fields within jets is not well understood. Flux conservation would cause the fields to stretch and weaken as they propagate outward, but at least in some powerful jets the fields are amplified during the jet propagation (Begelman et al. 1984). We ignore the subtleties involved in the jet evolution and simply assume that, at the end of the jet phase, the bubble has magnetic energy equal to a fixed fraction of the injected thermal energy, so that $E_{B}=\epsilon_{B} E_{K}$. The value of $\epsilon_{B}$ is highly uncertain, and we will regard it as a free parameter (see $\S 3$ ). The geometry of the field is also unclear; in radio-weak jets the transverse field dominates far from the core, but the opposite is true in radio-strong jets (Bridle \& Perley 1984) because of the aforementioned field amplification processes within such jets. We therefore assume for simplicity that the field is tangled on small scales and that it affects the shell dynamically only through an isotropic pressure component $p_{B}=(1 / 3) B^{2} / 8 \pi$.

Our prescription implicitly assumes that the magnetized plasma ejected from the quasar mixes efficiently with the ambient thermal gas. Such mixing has been inferred in some systems. For example, Bicknell, Cameron, \& Gingold (1990) showed that the fluctuating Faraday rotation signal observed in Cygnus A and PKS 2104-25N may be due to a region in which the magnetic field of the radio source has mixed with entrained ambient gas through turbulence excited by the Kelvin-Helmholtz instability on the surface of the radio lobes. The prescription also assumes that the field is mixed and randomly oriented on small scales. Unfortunately, little is known of the evolution of the fields in extended radio sources. Dreher et al. (1987) and Taylor et al. (1990) examined synchrotron polarization from the radio lobes of Cygnus A and Hydra A. They found the prevailing magnetic fields to be oriented parallel to the edge of the lobe. However, it is unclear whether this apparent coherence is due to large-scale regular fields or to small-scale, tangled fields that have developed anisotropies through shear with the external medium (Laing 1980). We expect that, even if the field is coherent on large scales in the jets or lobes, the turbulent mixing that occurs as the radio lobe entrains ambient thermal gas will break any large-scale coherence of the fields. The model of Bicknell et al. (1990) requires that such mixing occur on the scale of the KelvinHelmholtz surface waves generated between the lobe and the ambient medium. In this model, the fluctuating Faraday rotation measures on scales much smaller than the radio lobes indicate that such a mixing process will break any large-scale field coherence. Note that, in our scenarios, the magnetic fields do not dominate the expansion of the remnant bubbles (see Fig. 3 below); therefore, the issue of how the fields evolve is not critical for much of the following discussion.

In BAL quasars, the geometry of the outflow and its covering fraction are unknown. In $\S 2.1 .4$ we will show that the extreme assumption of a spherically symmetric outflow during the early phase results in only a small decrease in the final radius relative to the jet case. Because models suggest that $f_{c} \sim 0.1$ in BAL quasars (Weymann 1997), so that the outflow is closer to being collimated than to being isotropic, we use the jet propagation algorithm in our calculations for BAL quasars as well as for RLQs with only small errors.

\subsubsection{Late Expansion}

The isotropization of the energy burst from the quasar results in an overpressured shell embedded within a smooth, lower pressure environment. The shell expands, sweeping up ambient matter, until its velocity matches the Hubble flow (Tegmark et al. 1993) or until the binding gravitational potential of the halo causes the shell to recollapse. The equation of motion of the shell, with radius $R$ 
and mass $M_{s}$, is

$$
\begin{aligned}
\ddot{R}= & \frac{4 \pi R^{2}}{M_{s}}\left(p_{T}+p_{B}-p_{\mathrm{ext}}\right) \\
& -\frac{G}{R^{2}}\left[M_{d}(R)+M_{\mathrm{gal}}+\frac{M_{s}}{2}\right] \\
& +\Omega_{\Lambda}(z) H^{2}(z) R-\frac{\dot{M}_{s}}{M_{s}}\left[\dot{R}-v_{p}(R)\right],
\end{aligned}
$$

where $H(z)=H_{0} h(z)=H_{0}\left[\Omega_{0}(1+z)^{3}+\Omega_{\Lambda 0}\right]^{1 / 2}, \Omega_{\Lambda}(z)=$ $\Omega_{\Lambda 0}\left[\Omega_{0}(1+z)^{3}+\Omega_{\Lambda 0}\right]^{-1}, p_{T}$ is the thermal pressure of the bubble interior, $p_{\text {ext }}$ is the pressure of the external medium, $M_{d}(R)$ is the mass of dark matter interior to the shell, $M_{\mathrm{gal}}$ is the mass of baryonic matter that has cooled onto the galaxy, and $\dot{M}_{s}$ is the rate at which mass is swept into the shell (see below). Here the first term describes the pressure gradient across the shell, the second term describes the gravitational attraction between the halo and the shell (and the self-gravity of the shell), and the third term describes the effect of the cosmological constant, which amounts to a fraction $\Omega_{\Lambda 0}$ of the critical density at present. The final term describes the drag force from accelerating the swept-up matter to the bubble velocity.

As the shell travels outward it sweeps up ambient matter at a rate

$$
\dot{M}_{s}= \begin{cases}0 & v_{p}(R) \geq \dot{R} \\ 4 \pi R^{2} \rho_{g}(R)\left[\dot{R}-v_{p}(R)\right] & v_{p}(R)<\dot{R} .\end{cases}
$$

Flux conservation of the tangled magnetic field during the expansion of the shell implies

$$
p_{B}(R)=p_{B}\left(R_{i}\right)\left(\frac{R_{i}}{R}\right)^{4},
$$

where $R_{i}$ is the initial radius of the shell. We implicitly assume here that the magnetic field is frozen into the expanding bubble, i.e., that the bubble is ionized. In fact, the plasma in individual bubbles may recombine, after which ambipolar diffusion could separate the neutral gas from the magnetic field. However, we find that recombination in the bubbles does not occur before cosmological reionization (see §3.3), and so we can safely ignore this possibility.

The prescription of equation (12) also ignores any complications during the evolution (e.g., reconnection between flux tubes or flux conversion between toroidal and poloidal components). Reconnection will occur on a timescale $\tau \sim$ $L / v_{\mathrm{A}}$, where $L$ is the coherence length of the field and $v_{\mathrm{A}}$ is the Alfvén velocity. Our uncertainty in $L$ necessarily limits any treatment of reconnection. Fortunately, the magnetic pressure does not dominate the expansion of the bubble remnants, so such effects will not strongly modify the bubble evolution (see Fig. 3 below). Our prescription for the magnetic energy input guarantees that $p_{B} / p_{T}=1 / 20$ initially. Because $p_{B} / p_{T} \propto R$ (neglecting cooling), the magnetic pressure will increase in importance as the bubble expands; nevertheless, we find that magnetic pressure does not come to dominate any of the bubbles before the phase of rapid expansion ends.

Processes such as reconnection may affect the total magnetic energy in the bubble: reconnection, for example, will convert magnetic energy to thermal energy in the plasma. On the largest scales $(L \geq 100 \mathrm{kpc})$, we do not expect this effect to be strong because for such coherence lengths the reconnection time $L / v_{\mathrm{A}} \gg H_{0}^{-1}$ for reasonable field strengths at the mean cosmological density. Nevertheless, it is an issue that certainly merits further study because reconnection of tangled fields on small scales could potentially further decrease the remnant magnetic field strength.

The thermal pressure decreases as a result of both expansion and energy losses (Tegmark et al. 1993):

$$
\dot{p}_{T}=\frac{\Lambda}{2 \pi R^{3}}-5 p_{T} \frac{\dot{R}}{R} .
$$

Here $\Lambda$ is the complete heating/cooling function, which includes several components due to different physical processes inside the bubble interior,

$$
\Lambda=L_{\mathrm{diss}}-L_{\mathrm{brem}}-L_{\text {comp }}-L_{\mathrm{ion}} .
$$

The second and third terms account for radiative cooling: $L_{\text {brem }}$ describes cooling due to bremsstrahlung emission, and $L_{\text {comp }}$ describes inverse Compton cooling off the cosmic microwave background.

In our model, the ambient medium is swept up and accelerated to the shell expansion speed through inelastic collisions with the shell material. $L_{\text {diss }}$ describes the fate of the energy dissipated in this process. The energy could be either radiated away within the shell (e.g., during shock cooling) or injected into the bubble interior through turbulence. We let $f_{d}$ be the fraction of the total kinetic power that is injected as heat into the interior,

$$
L_{\mathrm{diss}}=\frac{1}{2} f_{d} \dot{M}_{s}\left[\dot{R}-v_{p}(R)\right]^{2} .
$$

For a fully radiative blast wave, $f_{d}=0$ (Ostriker $\&$ McKee 1988). For an energy-conserving (adiabatic) blast wave, the amount of energy injected into the interior may be estimated in the following manner. Consider a uniform shell (of thickness $l \ll R$ ) sweeping up matter at a shock as it travels through an ambient medium of density $\rho_{0}$. Let the material inside the shell have a pressure $P_{1}$ and a mass density $\rho_{1}$. Inside the cavity created by the shell, there is a rarefied, hot bubble of approximately uniform pressure $P_{2}$. If we assume that the shell retains most of the swept-up matter, then the shock jump conditions $\left(\rho_{1}=4 \rho_{0}\right)$ and mass conservation imply that for a stationary ambient medium, $l \approx R / 12$. In the cosmological case, the shell is even thinner (Ostriker \& McKee 1988; Ikeuchi, Tomisaka, \& Ostriker 1983). At the inner boundary of the shell, $P_{1} \approx P_{2}$. Hence, the ratio between the thermal energy of the bubble cavity, $E_{2}$, and the thermal energy of the shell itself, $E_{1}$, is

$$
\frac{E_{2}}{E_{1}}=\frac{P_{2} V_{2}}{P_{1} V_{1}} \gtrsim 4
$$

where $V_{1}$ and $V_{2}$ are the volumes of the shell and cavity, respectively. Thus, $E_{2} /\left(E_{1}+E_{2}\right) \gtrsim 80 \%$ of the blast wave energy resides in the cavity, and $f_{d} \gtrsim 0.8$ for an adiabatic blast wave.

In order to identify whether the shells are adiabatic or radiative, we consider the effect of cooling just behind the shock front. The shock is initially strong, so that the postshock density is $\rho_{s}=4 \rho_{g}(R)$ and the postshock temperature is $T_{s}=\left(3 \mu m_{p} v_{s}^{2}\right) /(16 k)$, where $v_{s}=R-v_{p}$ and $k$ is Boltzmann's constant. The cooling time for this region is

$$
\tau_{c}=\frac{3 \mu_{b} m_{p} k T_{s}}{\rho_{s} \Lambda_{s}\left(T_{s}\right)}
$$


assuming that the gas is fully ionized. Here $\mu_{b} m_{p}$ is the mean mass of the ions and $\Lambda_{s}$ is the cooling function of the shocked material within the shell. We use the zerometallicity cooling function compiled by Sutherland \& Dopita (1993), with the addition of Compton cooling. When the age of the bubble exceeds $\tau_{c}$, we assume that the bubble makes an instantaneous transition from an adiabatic $\left(f_{d}=\right.$ $0.8)$ to a radiative $\left(f_{d}=0\right)$ phase. Our calculations show that the intergalactic bubbles begin as adiabatic blast waves and remain so over a substantial fraction of their subsequent expansion (see Voit 1996).

We find that a fixed value of $f_{d}=0.8$ for the entire bubble history is an adequate approximation, and we adopt this value throughout. For very high redshift quasars $\left(z_{0} \gtrsim 15\right)$, this prescription overestimates the final bubble size by $\sim 10 \%$; however, the error decreases with decreasing $z_{0}$ and is negligible for $z_{0} \lesssim 5$. This reflects the fact that Compton cooling dominates within the shell, so that $\Lambda_{s} \propto\left(1+z_{0}\right)^{4}$. Quasars at very high redshifts make only small contributions to our final results so the errors introduced by fixing $f_{d}=0.8$ are small. Because $f_{d}$ has a relatively strong effect on the final radius (see $\S 2.1 .4$ ), and because the blast wave could in principle have a shorter cooling time due to clumpiness, we also show some results for $f_{d}=0$.

The strong shock surrounding the expanding bubble could also accelerate cosmic rays and relativistic electrons (Blandford \& Eichler 1987), similarly to supernova remnants (Koyama et al. 1995; Tanimori et al. 1998). These accelerated particles would remain in the bubble interior and provide an extra nonthermal pressure force on the shell, although this pressure would be reduced if the relativistic cosmic rays leak out from the interior. If leakage is small, such a pressure component could mimic a nonzero value of $f_{d}$ even at late times.

Finally, some or all of the swept-up material will be ionized. Stebbins \& Silk (1986) found that if the temperature $T>1.5 \times 10^{4} \mathrm{~K}$, hydrogen will be collisionally ionized; below this critical value the gas remains neutral. If $f_{d}=0.8$, the shock will ionize all of the incoming hydrogen provided that the postshock temperature exceeds this threshold. Even if this condition is not satisfied, the bubble interior may be hot, and so we follow the temperature evolution of the initially ionized bubble,

$$
T_{b}=\frac{p_{T}}{n_{b} k},
$$

where $n_{b}$, the particle number density inside the bubble, is

$$
n_{b}=\frac{3 \epsilon_{i} f_{m}}{4 \pi \mu_{b} m_{p}} \frac{M_{s}}{R^{3}} .
$$

Here $\epsilon_{i}=1$ (2) if the gas is neutral (ionized). We assume that a fraction $f_{m} \sim 0.1$ of the shocked shell material leaks into its interior; this is likely to be an overestimate, particularly at late times (Ostriker \& McKee 1988; Ikeuchi et al. 1983), but it has little effect on the results. The energy lost per unit time in ionizing the incoming hydrogen is then approximately

$$
L_{\mathrm{ion}}=E_{\mathrm{H}} \frac{f_{i} \dot{M}_{s}}{\mu_{b} m_{p}},
$$

where $E_{\mathrm{H}}=13.6 \mathrm{eV}$ is the ionization potential of hydrogen and

$$
f_{i}= \begin{cases}1 & f_{d}=0.8, z>z_{r}, T_{s}>1.5 \times 10^{4} \mathrm{~K} \\ 0.1 & z>z_{r}, T_{s}<1.5 \times 10^{4} \mathrm{~K}, \mathrm{~T}_{b}>1.5 \times 10^{4} \mathrm{~K} \\ 0 & \text { otherwise }\end{cases}
$$

is the fraction of swept-up material that is ionized. Note that if the universe has already been reionized at $z=z_{r}$, no ionization losses occur. In principle, once the bubble has expanded sufficiently, the temperature could decrease to a point at which hydrogen recombines. When this occurs, there will be a rapid pressure drop inside the bubble. However, we can neglect this effect because for nearly all bubbles in our model, recombination does not occur until well after the IGM has been reionized.

Our model requires knowledge of the external pressure as a function of redshift. Prior to reionization, the IGM around the quasar may recombine shortly after the quasar stops illuminating it with ionizing photons. In this case the bubble would propagate into a neutral IGM where the pressure is very low. The baryon temperature decreases adiabatically, $T_{\mathrm{IGM}} \propto(1+z)^{2}$ between the redshift of thermal decoupling from the microwave background $\left(z_{t} \approx\right.$ $100)$ and reionization (Barkana \& Loeb 2001). We assume that reionization occurs instantaneously at $z_{r}=7$ (Gnedin 2000 ) and that it raises the IGM temperature to $T_{\mathrm{IGM}} \approx 1-2$ $\times 10^{4} \mathrm{~K}$ subsequently. The IGM temperature continues to rise slowly until the present time, particularly in moderately overdense regions (Davé et al. 1999; Gnedin 2000). In modeling $z<z_{r}$, we adopt $p_{\text {ext }}=2 n(z) k T_{\mathrm{IGM}}$, where $T_{\mathrm{IGM}}=1$ $\times 10^{4} \mathrm{~K}$ and $n(z)$ is the mean cosmic baryon density. The effects of a higher ambient pressure are examined in $\S$ 2.1.4. Prior to reionization, the quasar photoionizes a large region of the IGM in its vicinity, and the shell will thus initially propagate through an ionized medium. In this case, the external pressure will not be negligible even at high redshifts; however, the source will also not lose any internal energy in ionizing the swept-up material. We find, like Barkana \& Loeb (2001), that these two effects nearly cancel, and so we ignore this subtlety.

Equations (10), (11), (12), and (13) fully describe the growth of each bubble.

For massive halos, the deep gravitational potential of the host galaxy prevents the shell from escaping and causes it to fall back into the virialized region. For $\epsilon_{B}=0.1, \tau_{q}=10^{7} \mathrm{yr}$, and $f_{d}=0.8$, we find that the maximum halo mass for which escape is possible is $M_{h, \max } \approx 2 \times 10^{13} M_{\odot}$ at $z=3$ and $M_{h, \max } \approx 3 \times 10^{11} M_{\odot}$ at $z=15$. We do not include halos more massive than this in our calculation; their magnetized bubbles do not experience any cosmological expansion and therefore make only a small contribution to the volume filling factor of magnetized regions. The shells in less massive halos continue to expand in physical coordinates until the present day. We halt such shells at the point of their maximum expansion in comoving coordinates.

\subsubsection{Parameter Dependence}

A simple, and surprisingly accurate, estimate of the final bubble radius can be obtained by noting that the mechanical (plus magnetic) quasar energy $E_{0}$ will essentially accelerate all the matter it encounters into a thin shell at a comoving distance $\hat{R}_{\max }=R_{\max }(1+z)$ from the central source. In doing so, it must accelerate this material to the Hubble flow velocity at that distance, $v=H\left(z_{0}\right) R_{\max }$. (Here 
we assume that the bubble reaches its maximum comoving size quickly compared to the age of the universe; see below for a discussion of the importance of this assumption.) At the onset of shell expansion, there are two energy reservoirs: the input energy of the quasar itself, $E_{0}$, and the kinetic energy of the preexisting Hubble flow within a distance $R_{\max }$ of the quasar, $(3 / 10) M_{s} v^{2}$. If the shell is radiative, the Hubble flow energy is lost during shock cooling and is unavailable in expanding the shell. If the expansion is adiabatic, a fraction $f_{d} \sim 0.8$ of the Hubble flow energy remains in the bubble and contributes to the expansion. Therefore, if we neglect the gravitational deceleration of the host halo as well as cooling inside the bubble cavity, energy conservation implies $E_{0} \approx K M_{s} v^{2} / 2$, where $K=0.52$ if $f_{d}=0.8$ and $K=1$ if $f_{d}=0$. Equivalently,

$$
\begin{aligned}
\hat{R}_{\max } \approx & \frac{1.3}{K^{1 / 5}}\left\{\left[\frac{1+z_{0}}{h\left(z_{0}\right)}\right]\left(\frac{0.7}{h}\right)\right\}^{2 / 5} \\
& \times\left\{\left(\frac{\epsilon_{\mathrm{bol}}}{0.093}\right)\left(\frac{\epsilon_{h}}{4 \times 10^{-4}}\right)\left[\frac{\epsilon_{K}\left(1+\epsilon_{B}\right)}{1.1}\right]\right\}^{1 / 5} \\
& \times\left[\left(\frac{0.019}{\Omega_{b} h^{2}}\right)\left(\frac{\tau_{q}}{10^{7} \mathrm{yr}}\right)\left(\frac{M_{h}}{10^{10} M_{\odot}}\right)\right]^{1 / 5} \mathrm{Mpc} .
\end{aligned}
$$

Note that the resulting $\hat{R}_{\max }$ is only a weak function of $E_{0} \propto \epsilon_{K}\left(1+\epsilon_{B}\right) \tau_{q} M_{h}$ and so is rather robust to changes in our model parameters.

We can also estimate the maximum halo mass for which the escape of the bubble shell is possible by comparing the total gravitational potential energy of the gas in an NFW halo (out to $r=\infty$ ) to the total energy output of the quasar plus the Hubble flow kinetic energy of the swept-up medium, if it is not radiated away through shock cooling. The result is that the shell can escape if $M_{h} \lesssim M_{\text {esc }}$, where

$$
\begin{aligned}
M_{\mathrm{esc}}=\frac{1.4}{} \times 10^{13} & K^{3 / 2} \\
& \times\left\{\left(\frac{\epsilon_{\mathrm{bol}}}{0.093}\right)\left(\frac{\epsilon_{h}}{4 \times 10^{-4}}\right)\left[\frac{\epsilon_{K}\left(1+\epsilon_{B}\right)}{1.1}\right]\left(\frac{\tau_{q}}{10^{7}}\right)\right\}^{3 / 2} \\
& \times\left[\left(\frac{1+z_{0}}{11}\right)\left(\frac{\Omega_{b} h^{2}}{0.019}\right)\right]^{-3 / 2} \\
& \times\left[\frac{\Omega_{0}}{\Omega\left(z_{0}\right)}\right]\left(\frac{\Delta_{c}}{200}\right)^{-1 / 2} g(c)^{-3 / 2} M_{\odot}
\end{aligned}
$$

In this expression, $g(c)$ is a function of the concentration parameter of the halo (which must be calculated numerically for each halo; see eq. [3]):

$$
g(c)=\frac{c}{[\ln (1+c)-c /(1+c)]^{2}} .
$$

The above expression provides a crude estimate of the halo mass above which gravity dominates the shell dynamics. At $z=15$ it overestimates the actual threshold mass by a factor of $\sim 4$; at $z=5$ it underestimates this mass by a factor of $\sim 2$.

Figure 1 shows the final $(z=0)$ bubble radius $\hat{R}$ as a function of halo mass. The solid curve shows the results of our numerical model, with $f_{d}=0.8$, while the dotted line is calculated from equation (22) with $K=0.52$ for $M<M_{\text {esc }}$. Both curves assume $z_{0}=10, \tau_{q}=10^{7} \mathrm{yr}$, and $\epsilon_{B}=0.1$. The agreement is surprisingly good. Over most of the mass

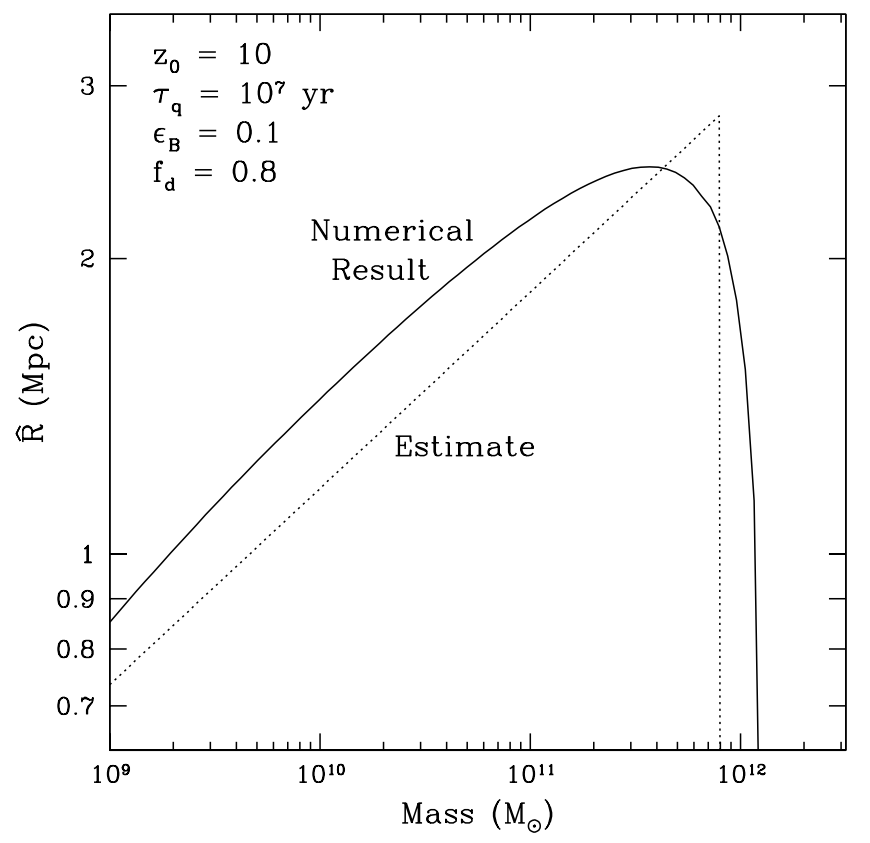

Fig. 1. - Final $(z=0)$ bubble radius $\hat{R}$ as a function of halo mass. The solid line shows the results of our numerical model with $f_{d}=0.8$, while the dotted line shows the estimate of eq. (22) with $K=0.52$ and a mass cutoff determined by eq. (23). Both curves assume standard parameter values, with $z_{0}=10, \tau_{q}=10^{7} \mathrm{yr}$, and $\epsilon_{B}=0.1$.

range, equation (22) underestimates the final comoving radius by only $\sim 20 \%$. This accuracy is in fact due to a fortuitous cancellation of two errors in our estimate. First, we neglect cooling within the bubble cavity, the external pressure of the IGM, and the gravitational potential of the host halo. For these reasons we would expect equation (22) to overestimate the final comoving radius. On the other hand, the estimate neglects cosmological deceleration. As the age of the universe increases, the Hubble flow decelerates (until the cosmological constant begins to dominate at $z \sim 1$ ). Therefore, the Hubble flow velocity at a fixed comoving distance from the host decreases with decreasing redshift. Because the mass within a given comoving radius is fixed, less energy is required in order to accelerate the shell to a given comoving distance at smaller redshifts. Equation (22) assumes that the shell reaches its maximum radius instantaneously, while in reality the shell continues to expand for many Hubble times (see Fig. 3 below). For this reason, we would expect it to underestimate the final radius. Figure 1 shows that these two errors nearly cancel. Voit (1996) constructed an analytic self-similar solution to the expansion of a blast wave in an $\Omega_{\Lambda}=0$ universe that included the effects of cosmological deceleration. Barkana \& Loeb (2001), using a numerical model similar to ours, show that this solution overestimates the true size of the blast waves by a factor of $\sim 2$ because it neglects cooling, external pressure, and the gravity of the host halo. Evidently, ignoring cosmological deceleration gives a factor of $\sim 2.5$ underestimate, and so our estimate is only $\sim 20 \%$ smaller than the true bubble size.

Figure 2 shows the numerical solution for the comoving bubble radius $\hat{R}$ as a function of redshift for several different examples. All cases assume that $M_{h}=10^{10} M_{\odot}, z_{0}=10$, $\tau_{q}=10^{7} \mathrm{yr}$, and $\epsilon_{B}=0.1$. Results are shown for our standard scenario (solid line), a case in which the gas in the halo of the host galaxy does not cool (short-dashed line), a case in 


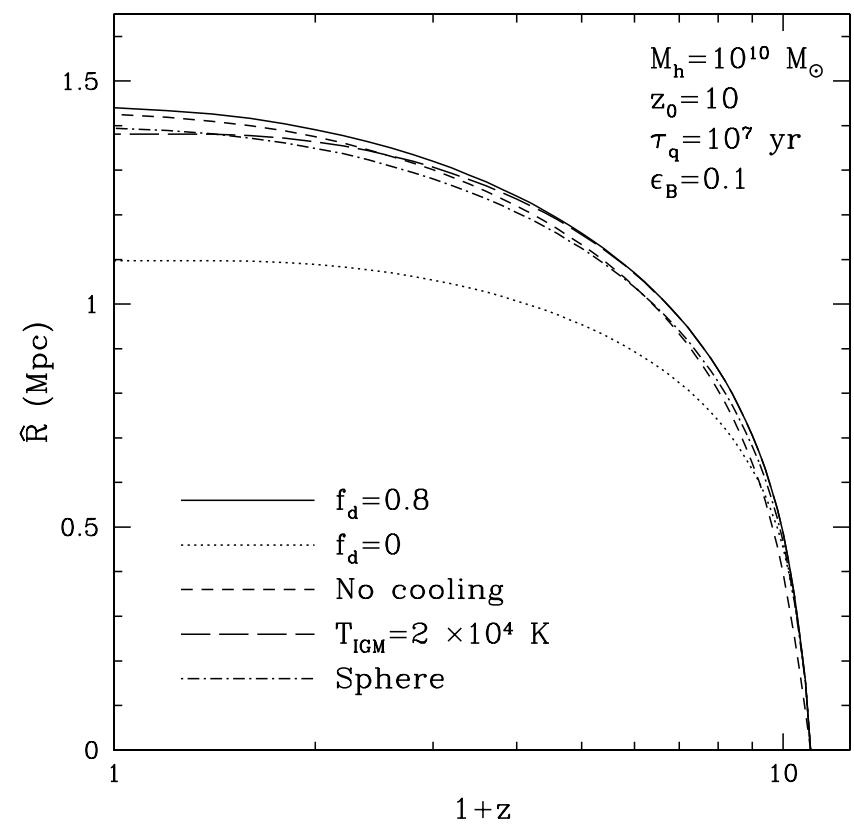

FIG. 2.-Comoving bubble radius $\hat{R}$ as a function of redshift for a variety of scenarios. All cases assume $M_{h}=10^{10} M_{\odot}, z_{0}=10, \tau_{q}=10^{7} \mathrm{yr}$, and $\epsilon_{B}=0.1$. Results are shown for our standard scenario (solid line), a case in which the gas in the halo of the host galaxy does not cool (short-dashed line), a case in which $T_{\mathrm{IGM}}=2 \times 10^{4} \mathrm{~K}$ after reionization (long-dashed line), and a case in which the outflow is initially spherical rather than a jet (dotdashed line); all of these assume $f_{d}=0.8$. Also shown is a case in which $f_{d}=0($ dotted line $)$.

which $T_{\mathrm{IGM}}=2 \times 10^{4} \mathrm{~K}$ after reionization (long-dashed line), and a case in which the outflow is initially spherical rather than a jet (dot-dashed line); all of these curves assume $f_{d}=0.8$. Also shown is a case in which $f_{d}=0$ (dotted line).

Clearly, the parameter that has the greatest effect on the final result is $f_{d}$. Varying any of our other assumptions makes a substantially smaller difference in our results. Although the initial expansion slows down considerably when the virialized gas does not cool, the late-phase expansion remains roughly the same because the swept-up mass is dominated by gas outside the virial radius, so that the final radius is only slightly smaller. To describe spherical expansion during the initial phase, we use the model of $\S 2.1 .3$, including an extra source term in the heating/cooling function for the quasar mechanical luminosity. This has a somewhat larger effect because plowing through the ambient medium during this phase is significantly more difficult than sweeping aside the matter in a jet beam. As a result, spherical flows decelerate more rapidly early on, decreasing the final bubble radius by $\sim 5 \%$.

Increasing the post-reionization temperature to the value typical of quasar reionization models, $T_{\mathrm{IGM}}=2 \times 10^{4} \mathrm{~K}$, decreases the final radius by $\sim 5 \%$. In any reionization model, the temperature of the IGM is expected to continue to rise until the present day in moderately overdense regions (Davé et al. 1999). This is particularly important as groups of galaxies form at $z \lesssim 2$; the gas between the group galaxies will be at the virial temperature of the group, so outflows from quasars embedded in these systems must propagate through a hot, high-density medium. This pressure increase could suppress the filling factor of magnetized regions from low- $z$ quasars, but it has little effect on the high- $z$ contribution because the contributing halos will have already expanded to be very close to their maximum comoving size by the time the mean IGM temperature increases substantially. The effects of an increase in the external pressure will be discussed further in $\S 3.1$.

Figure 3 shows the comoving bubble radius $\hat{R}$ as a function of redshift for quasars that produce an outflow at different redshifts $z_{0}$. In all cases $\tau_{q}=10^{7} \mathrm{yr}$ and $f_{d}=0.8$. The solid curves assume $M_{h}=10^{10} M_{\odot}$ and $\epsilon_{B}=0.1$, with $z_{0}=$ $20,15,10,5$, and 3 , from right to left. The dotted curves show analogous results for $M_{h}=10^{10} M_{\odot}$ and $\epsilon_{B}=0$. The dashed curve shows the bubble radius for $M_{h}=3 \times 10^{12}$ $M_{\odot}$ and $z_{0}=10$, illustrating the evolution of a quasar outflow in a massive halo in which the gravitational potential suppresses the expansion into the IGM. As expected from equation (22), older quasars produce smaller bubbles. This is simply a result of cosmological deceleration: highredshift outflows join the Hubble flow earlier, when the expansion speed for a fixed comoving distance is larger. Therefore, accelerating a shell to a fixed comoving size requires a more energetic outflow (or a more massive halo, according to our prescription) at higher redshifts. Note that the $z_{0}=3$ quasar has not yet approached its asymptotic final radius. The small differences between the solid and dotted curves confirm the prediction of equation (22), $\hat{R}_{\max } \propto\left(1+\epsilon_{B}\right)^{1 / 5}$. For all reasonable values of the magnetic energy parameter $\epsilon_{B}$, the final bubble radius is insensitive to the particular value chosen.

\subsection{Source Populations}

The most easily detectable type of quasar outflow is a radio jet. Unfortunately, the relation between the radio power and kinetic jet power is complicated and highly uncertain (Begelman et al. 1984; Willott et al. 1999), and so it is difficult to infer a kinetic luminosity function from the

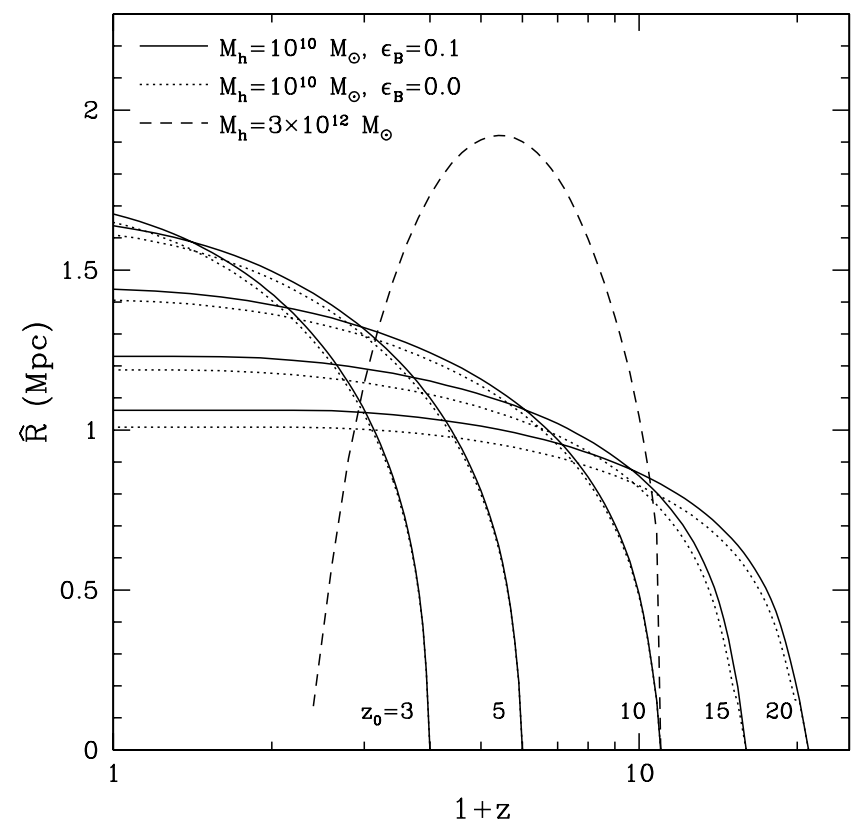

FIG. 3.-Comoving bubble radius $\hat{R}$ as a function of redshift for quasars that produce an outflow at different redshifts, $z_{0}$. For the solid curves, $M_{h}=10^{10} M_{\odot}$ and $\epsilon_{B}=0.1$. Beginning from right to left, $z_{0}=20$, $15,10,5$, and 3. The dotted curves show analogous solutions for $M_{h}=10^{10}$ $M_{\odot}$ and $\epsilon_{B}=0$. The dashed curve shows the radius for $M_{h}=3 \times 10^{12} M_{\odot}$ and $z_{0}=10$ and illustrates the recollapse of a bubble for a massive halo in which gravity prevents escape into the IGM. In all cases, $\tau_{q}=10^{7} \mathrm{yr}$ and $f_{d}=0.8$. 
radio luminosity function of quasars. In addition, other forms of outflows, such as those in BAL quasars, may not be radio loud. On physical grounds, one would expect the kinetic luminosity to carry some fraction of the total accretion energy of the quasar (e.g., Blandford \& Begelman 1999), the rest of which is carried by radiation emanating from the inner region of the accretion flow. We therefore calibrate the kinetic luminosity of a quasar based on its bolometric luminosity, which in turn can be inferred (through the appropriate $k$-correction for the universal spectrum of Elvis et al. 1994) from its rest-frame $B$-band luminosity (see $\S 2.1 .1$ ).

For the optical luminosity function of quasars, we adopt an empirical parametrization based on observations at low redshifts $(z \lesssim 4)$ and extrapolate it to higher redshifts using a simple theoretical model. Pei (1995) presents two fits to the observed luminosity function at low redshifts: the first is a double power law, which provides a divergent contribution as the quasar luminosity approaches zero, and the second is a modified Schechter function, which converges for small luminosities. Because small halos have a large effect on the results, we conservatively use the latter form. We modify equation (9) of Pei (1995) to our chosen cosmology in order to describe the quasar abundance as a function of halo mass and redshift, $\Phi\left(M_{h}, z\right)$. Since $\tau_{q} \ll H(z)^{-1}$, the formation rate per comoving volume of quasars with outflows is

$$
\frac{d n_{q}}{d M_{h} d z} \approx f^{\mathrm{Pei}} \frac{\Phi\left(M_{h}, z\right)}{\tau_{q}}\left|\frac{d t}{d z}\right|, \quad z \leq 4,
$$

where $f^{\mathrm{Pei}}$ is the fraction of quasars with magnetized outflows.

We extrapolate the quasar luminosity function to high redshifts, $z \gtrsim 4$, using the Press-Schechter mass function of halos (Press \& Schechter 1974). Previous studies have shown that under the assumptions of a universal quasar lifetime and a universal quasar light curve, this approach can reproduce the observed quasar $B$-band luminosity function at $2 \lesssim z \lesssim 5$ (Haiman \& Loeb 1998). The number of quasars with outflows per comoving volume forming in each host mass range at a redshift $z$ is then

$$
\frac{d n_{q}}{d M_{h} d z} \approx f^{\mathrm{PS}} \frac{d}{d z}\left(\frac{d n_{\mathrm{PS}}}{d M_{h}}\right), \quad z>4,
$$

where $d n_{\mathrm{PS}} / d M_{h}$ is the Press-Schechter mass function and $f^{\mathrm{PS}}$ is the fraction of these halos hosting magnetized outflows.

Matching the two formation rates at $z \approx 4$ yields the relation

$$
f^{\mathrm{Pei}}=f^{\mathrm{PS}}\left(\frac{\tau_{q}}{10^{6} \mathrm{yr}}\right)
$$

The most natural choice is $\tau_{q}=10^{6}$ yr and $f^{\mathrm{PS}}=f^{\mathrm{Pei}}$, as shown by Haiman \& Loeb (1998). However, we adopt $\tau_{q}=$ $10^{7} \mathrm{yr}$ and $f^{\mathrm{PS}}=0.1 f^{\mathrm{Pei}}$ as our standard values for two reasons. First, spectral aging of radio lobes indicates ages of $\sim(1-3) \times 10^{7}$ yr (Kaiser 2000). Second, a choice of quasar lifetime fixes the total number of quasars, and hence remnant black holes, per comoving volume. Choosing $\tau_{q}=$ $10^{7} \mathrm{yr}$ yields a present-day black hole mass density $\rho_{\mathrm{BH}} \sim 2$ $\times 10^{6} M_{\odot} \mathrm{Mpc}^{-3}$. Recent estimates, based on the proportionality between black hole and bulge mass (Magorrian et al. 1998; Gebhardt et al. 2000), have found $\rho_{\mathrm{BH}}^{\text {obs }} \sim 7 \times 10^{5}$
$M_{\odot} \mathrm{Mpc}^{-3}$ (Salucci et al. 1999). Because $\rho_{\mathrm{BH}} \propto \tau_{q}^{-1}$, decreasing the lifetime below this level will lead to an unacceptably large mass in remnant black holes. Our black hole density is still somewhat larger than most observational estimates; therefore, we also show some results for $\tau_{q}=10^{8}$ $\mathrm{yr}$ and $f^{\mathrm{PS}}=0.01 f^{\mathrm{Pei}}$ below. By prescribing a larger total accretion efficiency, this choice yields a remnant black hole density close to that normally assumed for optical quasars (Chokshi \& Turner 1992).

The minimum halo mass to host a quasar, $M_{h, \min }$, is determined by the criteria for efficient cooling and infall. Before reionization, the lack of metals in the primordial gas prohibited efficient cooling unless the virial temperature $T_{v}>10^{4} \mathrm{~K}$, so as to allow atomic excitations and cooling via line radiation (Barkana \& Loeb 2001). After reionization, the increased pressure due to photoionization heating increases the Jeans mass, suppressing infall onto low-mass galaxies. This process prevented the gas from collapsing into halos with a circular velocity $v_{c} \lesssim 50 \mathrm{~km} \mathrm{~s}^{-1}$ (Efstathiou 1992; Navarro \& Steinmetz 1997; Thoul \& Weinberg 1996). We also allow for the possibility of a lowmass cutoff for the hosts of quasars with strong outflows. Laor (2000) has argued for such a relation in RLQs on empirical grounds, although there is no firm physical theory for the existence of such a cutoff.

\section{RESULTS}

In most of our calculations we assume $\epsilon_{B}=0.1$ and $f_{R}^{\mathrm{Pei}}=0.1$, the latter being the typical fraction of RLQs (Stern et al. 2000). We refer to this as the RLQ model. The magnetic energy fraction in radio jets is highly uncertain; estimates are commonly based upon an assumption of equipartition, although there is a notorious lack of observational data to support this assumption (Begelman et al. 1984). At equipartition, the total energy is shared between hydrodynamic motions, thermal protons, thermal electrons, accelerated cosmic rays, and magnetic fields, yielding $\epsilon_{B} \lesssim$ 0.2 .

It is quite possible that the winds in BAL quasars also carry a substantial magnetic energy fraction, especially since some models predict that the BAL wind is powered by magnetic forces (e.g., de Kool \& Begelman 1995). For illustrative purposes, we discuss a model with $f_{\mathrm{BAL}}^{\mathrm{Pei}}=1.0$ and $\epsilon_{B}=0.01$; we refer to this scenario as the BALQSO model. The $\sim 10 \%$ fraction of BAL systems among all quasars is observationally found to be constant even at high redshifts (Storrie-Lombardi et al. 2001; Schneider et al. 2001) and reflects the covering fraction of their outflows in our model. The small value of $\epsilon_{B}$ is chosen so that the outflows in this model may also characterize the vast majority of all quasars that are radio quiet. We stress, however, that the value adopted for $\epsilon_{B}$ is highly uncertain in this case.

We use the above two models to bracket the range of interest; together, they allow us to discuss the physical implications of our scenario for magnetic field generation in the IGM. The RLQ model fills $\sim 20 \%$ of space with magnetized regions in which the magnetic energy and thermal energy of a photoionized IGM are near equipartition. Numerical simulations show that the structure of the Ly $\alpha$ forest can be reconstructed without including magnetic fields (Davé et al. 1999). Higher values of $\epsilon_{B}$ may therefore conflict with existing data. The BALQSO model fills nearly all of the IGM with magnetized regions, and so we adopt a small value of $\epsilon_{B}$ for it. 
Figures 4-7 show results for the RLQ model. Because the results scale simply with $\epsilon_{B}$ and $f^{\text {Pei }}$, we do not show analogous plots for the BALQSO model. For ease of display, we define $\epsilon_{-1} \equiv \epsilon_{B} / 0.1$ and $\widetilde{B} \equiv B /\left(\epsilon_{B} / 0.1\right)^{1 / 2}$.

\subsection{Filling Factor and Mean Magnetic Energy Density}

Assuming that the sources follow a Poisson distribution in space, the filling factor of their magnetized bubbles at redshift $z$ is $F(z)=1-e^{-\phi(z)}$, where

$$
\phi(z)=\int_{z}^{\infty} d z^{\prime} \int_{M_{h, \min }}^{M_{h, \max }} d M_{h} \frac{d n_{q}\left(M_{h}, z^{\prime}\right)}{d M_{h} d z^{\prime}} \hat{V}\left(z ; M_{h}, z^{\prime}\right) .
$$

Here $\hat{V}\left(z ; M_{h}, z^{\prime}\right)$ is the comoving volume at redshift $z$ of a bubble produced by a quasar forming at redshift $z^{\prime}$ in a host of mass $M_{h}$. The top panel of Figure 4 shows the filling factor $F(z)$ for various RLQ model scenarios. The solid curves assume $f_{d}=0.8$ and that $M_{h, \text { min }}$ is determined by atomic cooling considerations before reionization and infall suppression afterward (top curve), $M_{h, \min }=10^{9} M_{\odot}$ (middle curve), and $M_{h, \min }=10^{10} M_{\odot}$ (bottom curve). The dashed curve assumes $f_{d}=0$ and that $M_{h, \min }$ is determined by atomic cooling and infall suppression. All curves assume that $f_{R}^{\mathrm{PS}}=0.01, f_{R}^{\mathrm{Pei}}=0.1, \tau_{q}=10^{7} \mathrm{yr}$, and $\epsilon_{B}=0.1$. In all cases, we find that the filling fraction is very small at high redshifts but rises to a substantial value at moderate and low redshifts $(\sim 15 \%-40 \%$ at the present day).

It is straightforward to approximate this integral analytically for the low-redshift case of equation (25). Such an approximation allows us to examine the dependence of the result on the model parameters. For this estimate, we use equation (22) for the bubble radius and neglect the high and

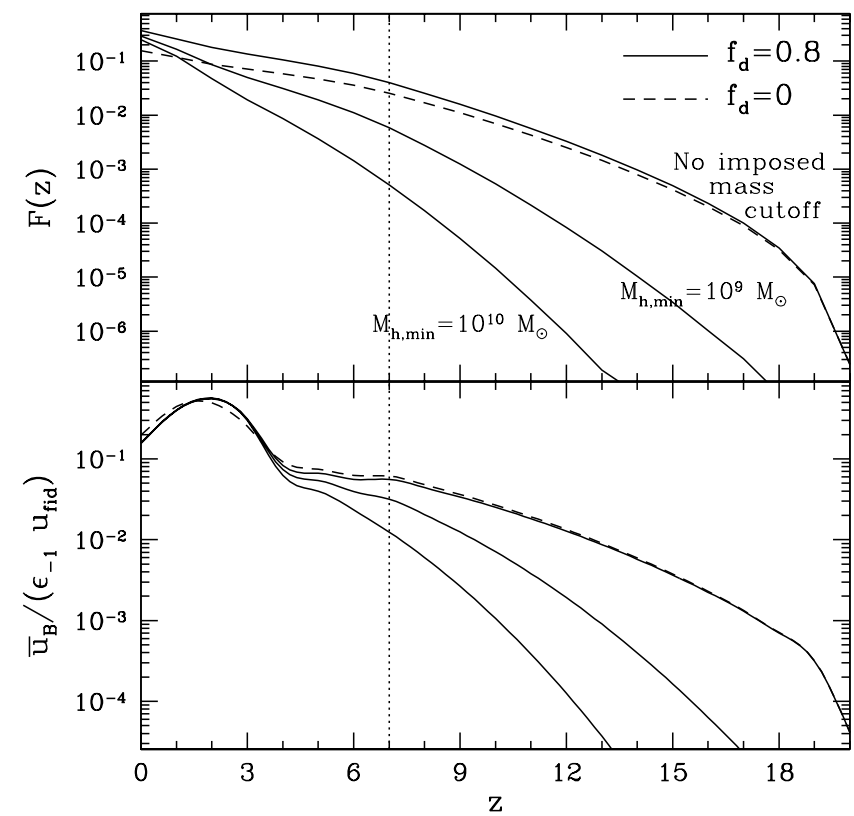

Fig. 4.-Top panel: Volume filling fraction of magnetized bubbles $F(z)$, as a function of redshift, for the RLQ model. Bottom panel: Ratio of normalized magnetic energy density, $\bar{u}_{B} / \epsilon_{-1}$, to the fiducial thermal energy density $u_{\mathrm{fid}}=3 n(z) k T_{\mathrm{IGM}}$, where $T_{\mathrm{IGM}}=10^{4} \mathrm{~K}$, as a function of redshift. In each panel, the solid curves assume $f_{d}=0.8$ and that $M_{h, \min }$ is determined by atomic cooling before reionization and infall suppression afterward (top curve), $M_{h, \min }=10^{9} M_{\odot}$ (middle curve), and $M_{h, \min }=10^{10} M_{\odot}$ (bottom curve). The dashed curve assumes $f_{d}=0$ and determines $M_{h, \min }$ by atomic cooling and infall suppression. The vertical dotted line indicates the assumed redshift of reionization, $z_{r}=7$. All curves assume $f_{R}^{\mathrm{PS}}=0.01$, $f_{R}^{\mathrm{Pei}}=0.1$, and $\tau_{q}=10^{7} \mathrm{yr}$. low halo mass cutoffs. The result of the integration is

$$
\begin{aligned}
\phi_{\mathrm{Pei}}(z) \approx & 0.3\left[\frac{G\left(z, z_{i}\right)}{0.29}\right]\left(\frac{f^{\mathrm{Pei}}}{0.1}\right)\left(\frac{\Phi_{*}}{10^{4.34} \mathrm{Gpc}^{-3}}\right) \\
& \times\left[\frac{\Gamma\left(32 / 5-4 \beta_{*}\right)}{4.48}\right]\left[\left(\frac{10^{7} \mathrm{yr}}{\tau_{q}}\right)\left(\frac{0.7}{h}\right)\right]^{2 / 5} \\
& \times\left\{\left(\frac{0.52}{K}\right)\left[\frac{\epsilon_{k}\left(1+\epsilon_{B}\right)}{1.1}\right]\left(\frac{L_{*}}{10^{9.78} L_{\odot}}\right)\right. \\
& \left.\times\left(\frac{0.019}{\Omega_{b} h^{2}}\right)\right\}^{3 / 5},
\end{aligned}
$$

where $G\left(z, z_{i}\right)$ is a function of the boundaries of the redshift interval over which quasars are considered:

$$
\begin{aligned}
G\left(z, z_{i}\right)= & \int_{z_{i}}^{z} d z^{\prime} \frac{\left(1+z^{\prime}\right)^{1 / 5}}{\left[\Omega_{0}\left(1+z^{\prime}\right)^{3}+\left(1-\Omega_{0}\right)\right]^{11 / 10}} \\
& \times \exp \left[\frac{-\left(z^{\prime}-z_{*}\right)^{2}}{10 \sigma_{*}^{2} / 3}\right],
\end{aligned}
$$

and $G(0,4)=0.29$. Quantities with an asterisk subscript are parameters from the Pei (1995) luminosity function: $\Phi_{*}$ is the space density normalization constant, $L_{*}$ characterizes the "break" luminosity between the power-law and exponential portions of the fit, $-\beta_{*}$ is the slope of the power-law portion, $z_{*}$ is the peak of the quasar era, and $\sigma_{*}$ characterizes the duration of the quasar era. (This luminosity function was derived for an open model with $\Omega_{0}=0.2$ and no cosmological constant. We must leave it in this form in order to evaluate the mass integral analytically, although our simulations do take place in a flat universe with $\Omega_{\Lambda 0}=$ 0.7.)

The analogous approximation for higher redshifts, when the source counts are given by equation (26), is less straightforward because of the nonanalytic form of the PressSchechter mass function. To begin, we fit a power law, $\sigma(M)=B\left(M / M_{\odot}\right)^{-\beta}$, to the fluctuation spectrum over the range $M=10^{8}-10^{10} M_{\odot}$, which is the mass interval most relevant for our calculation. With our standard cosmological parameters, the fluctuation spectrum is well fitted by $\beta=0.0826$ and $B=30.42$ in the range of interest. Then a numerical integration of equation (28), using equations (22) and (26), yields

$$
\begin{aligned}
\phi_{\mathrm{PS}}(z=0) \approx & 0.2\left\{\left(\frac{0.52}{K}\right)\left(\frac{\epsilon_{\mathrm{bol}}}{0.093}\right)\left(\frac{\epsilon_{h}}{4 \times 10^{-4}}\right)\right. \\
& \left.\times\left[\frac{\epsilon_{K}\left(1+\epsilon_{B}\right)}{1.1}\right]\left(\frac{\tau_{q}}{10^{7} \mathrm{yr}}\right)\left(\frac{0.019}{\Omega_{b} h^{2}}\right)\right\}^{3 / 5} \\
& \times\left(\frac{f^{\mathrm{PS}}}{0.01}\right)\left(\frac{\Omega_{0}}{0.3}\right)\left(\frac{h}{0.7}\right)^{4 / 5},
\end{aligned}
$$

where the dependence on $\Omega_{0}$ comes from the linear growth factor. (We have neglected the dependence of the growth factor on the cosmological constant; however, its effects will be negligible for the quasars at high redshifts to which we apply the formula.) In this estimate we assume that the minimum halo mass is determined by cooling before reionization and infall suppression afterward. Note the different dependence on $f$ and $\tau_{q}$ between this expression and the low-redshift result, equation (29): $\phi_{\mathrm{Pei}} \propto f^{\mathrm{Pei}} \tau_{q}^{-2 / 5}$, while $\phi_{\mathrm{PS}} \propto f^{\mathrm{PS}} \tau_{q}^{3 / 5}$. This is a direct result of the matching pro- 
cedure between the two source counts described by equation (27).

Figure 5 shows the relative contributions of high- and low-redshift quasars for several different choices of $\tau_{q}$ in the RLQ scenario, calculated with our full numerical model. The top panel shows separately the filling fraction $F(z)$ from quasars with source counts determined by equation (26) at $z_{0}>4$ and by equation (25) at $z_{0}<4$. We show results for $\tau_{q}=10^{7}$ (solid curves), $10^{8}$ (dashed curves), and $10^{6} \mathrm{yr}$ (dotdashed curves). In each case, $f_{R}^{\mathrm{PS}}$ is determined by equation (27). All curves assume $f_{R}^{\mathrm{Pei}}=0.1, f_{d}=0.8, \epsilon_{B}=0.1$, and $z_{r}=7$. As suggested by equations (29) and (31), the contributions to the filling factor at $z=0$ are comparable for the two regimes. Note that for $z \lesssim 4$, the filling factor from high-z sources is approximately constant with time, indicating that most of the bubble remnants from this population have reached their maximum comoving radii by this point. Equations (29) and (31) are accurate to within a factor of $\sim 1.5$, despite the fact that they do not include proper cosmological parameters or mass cutoffs and that they ignore the detailed dynamics of the expanding bubbles.

This panel also shows that modifying the assumed quasar lifetime has only a relatively small effect on the final filling factor: both the low $z$ and high- $z$ total contributions to $F(z)$ vary by less than a factor of about 2 when the value of $\tau_{q}$ is changed by 2 orders of magnitude. In addition, regardless of the choice for $\tau_{q}$, both regimes contribute approximately equal filling fractions. Note that, because equations (29) and (31) ignore the high-mass cutoff at $M_{\text {esc }}$, the true scaling is not exactly that given by these estimates. Because this limit-

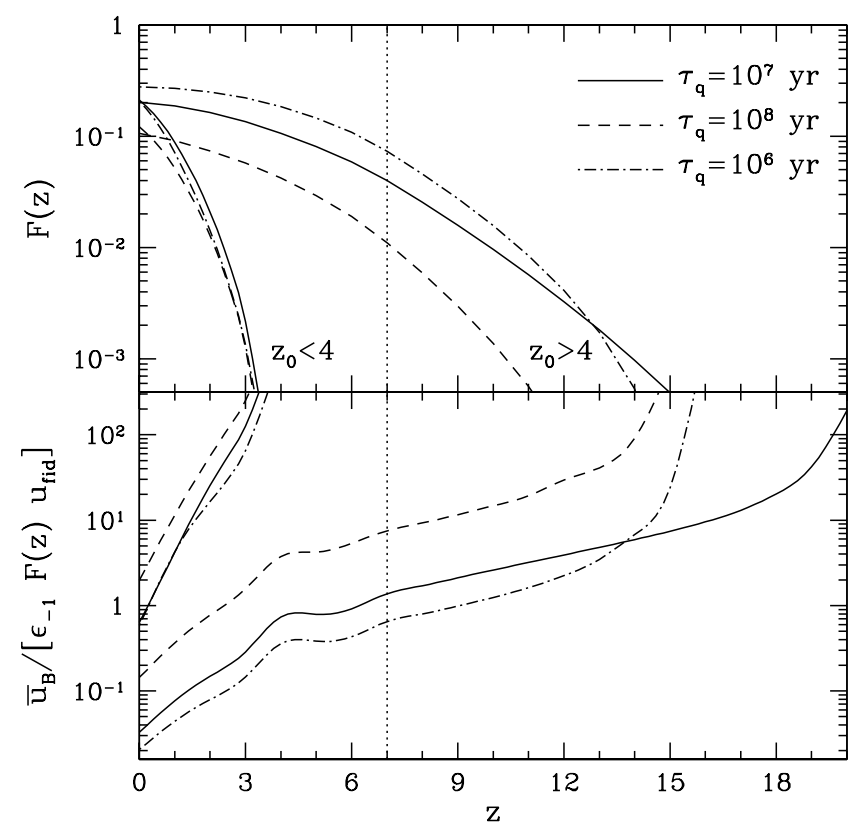

FIG. 5.-Top panel: Volume filling fraction of magnetized bubbles $F(z)$, as a function of redshift, for the RLQ model. Bottom panel: Ratio of magnetic energy per filled volume, $\bar{u}_{B} /\left[\epsilon_{-1} F(z)\right]$, to the fiducial thermal energy density $u_{\mathrm{fid}}=3 n(z) k T$, where $T=10^{4} \mathrm{~K}$, as a function of redshift. The set of lines beginning at the right indicates the contribution from high-redshift quasars calculated using the Press-Schechter formalism for $z_{0}>4$, while the set beginning at the left indicates the contribution determined from the Pei (1995) luminosity function for $z_{0}<4$. Results are shown for $\tau_{q}=10^{7}$ (solid curves), $10^{8}$ (dashed curves), and $10^{6} \mathrm{yr}$ (dotdashed curves), with $f_{R}^{\mathrm{PS}}$ determined by eq. (27). All curves assume $f_{R}^{\mathrm{Pei}}=0.1$ and $f_{d}=0.8$. The vertical dotted line indicates the assumed redshift of reionization, $z_{r}=7$. ing mass is a fairly strong function of the model parameters (e.g., $M_{\text {esc }} \propto \tau_{q}^{3 / 2}$; see eq. [23]), changing these parameters can sometimes have a dramatic effect on the results. This is particularly true at high $z$, when the minimum mass for efficient atomic cooling and the high-mass cutoff are close. For example, at $z=15$, the minimum mass for cooling is $\sim 10^{8} M_{\odot}$. If $\tau_{q}=10^{6}$ yr and $f_{d}=0.8$, then $M_{\text {esc }} \sim 5 \times 10^{9}$ $M_{\odot}$ at this redshift, while if $\tau_{q}=10^{7} \mathrm{yr}$ and $f_{d}=0.8$, then $M_{\text {esc }} \sim 3 \times 10^{11} M_{\odot}$. The shrinking of the allowed halo mass interval in the former case means that short-lifetime quasars are less efficient (by a factor $\sim 1.25$ ) than we would expect from the predicted scaling $\phi_{\mathrm{PS}} \propto f^{\mathrm{PS}} \tau_{q}^{3 / 5}$ of equation (31).

Equations (29) and (31) imply that the dependence of the filling factor $F(z)$ on the initial magnetic energy fraction is very weak, as $\phi \propto\left(1+\epsilon_{B}\right)^{3 / 5}$. The BALQSO model, which assumes that every quasar hosts a BAL outflow, is therefore more efficient at polluting large volumes than the RLQ model. Increasing the low-mass cutoff can dramatically decrease the contribution from high- $z$ sources because massive halos are considerably rarer at these epochs. Imposing a low-mass cutoff of $M_{h, \min }=10^{11} M_{\odot}$ reduces the filling factor from high- $z$ sources to $F_{\mathrm{PS}} \lesssim 10^{-3}$. However, such an increase in the low-mass cutoff has a much smaller effect on the low- $z$ contribution because the nonlinear mass scale continues to increase as the redshift decreases. In particular, in our standard RLQ scenario, sources with $M_{h} \geq 10^{11} M_{\odot}$ provide a present-day filling factor of $F_{\mathrm{Pei}}=0.16$, while sources with $M_{h} \geq 10^{12} M_{\odot}$ provide $F_{\mathrm{Pei}}=0.06$. Thus, even if only the most luminous quasars host outflows, a nonnegligible fraction of space can be polluted with magnetic fields. A quasar lifetime of $\tau_{q}=$ $10^{8} \mathrm{yr}$ increases the relative importance of these mass ranges by increasing the high-mass cutoff.

The global volume-averaged magnetic energy density is

$$
\begin{aligned}
\bar{u}_{B}(z)= & \int_{z}^{\infty} d z^{\prime} \int_{M_{h, \text { min }}}^{M_{h, \max }} d M_{h} \frac{d n_{q}\left(M_{h}, z^{\prime}\right)}{d M_{h} d z^{\prime}} \\
& \times \hat{V}\left(z ; M_{h}, z^{\prime}\right) u_{B}\left(z ; M_{h}, z^{\prime}\right),
\end{aligned}
$$

where $u_{B}\left(z ; M_{h}, z^{\prime}\right)$ is the mean (time-dependent) magnetic energy density at redshift $z$ inside a bubble produced by a quasar forming at redshift $z^{\prime}$ in a host of mass $M_{h}$. Note that the contribution of a single bubble $\propto \hat{V} u_{B}$, which decreases $\propto R^{-1}$ during the bubble's expansion. For reference, a typical bubble expands by a factor $\sim 10^{2}-10^{2.5}$ after the termination of the quasar's active phase if $\tau_{q}=10^{7} \mathrm{yr}$.

The bottom panel of Figure 4 shows the ratio between $\bar{u}_{B}(z) / \epsilon_{-1}$ and a fiducial value for the mean thermal energy density of the IGM,

$$
\begin{aligned}
u_{\mathrm{fid}}= & 3 n(z) k T_{\mathrm{IGM}}=6 \times 10^{-19}\left(\frac{\Omega_{b} h^{2}}{0.019}\right) \\
& \times\left(\frac{T_{\mathrm{IGM}}}{10^{4} \mathrm{~K}}\right)(1+z)^{3} \mathrm{ergs} \mathrm{cm}^{-3},
\end{aligned}
$$

where $T_{\mathrm{IGM}}=10^{4} \mathrm{~K}$ and $n(z)$ is the mean cosmic baryon density, for the same scenarios as shown in the top panel of Figure 4. We find that this ratio is approximately independent of $\epsilon_{-1}$. The above fiducial energy density was chosen only for normalization purposes. It corresponds to a uniform IGM that has been heated by a photoionizing radi- 
ation field, and hence it grossly overestimates the typical pressure in the neutral IGM before reionization $z \gtrsim 7-10$ (Gnedin \& Ostriker 1997; Gnedin 2000) and underestimates the temperature that the IGM acquires in large-scale shocks at low redshifts $z \lesssim 3$ (Cen \& Ostriker 1999; Davé et al. 1999). The signature of the "quasar era" is apparent in the figure; once the quasar abundance turns over, the mean magnetic energy density decreases as well.

Because the bubble size is nearly independent of the injected magnetic energy (eq. [22]), the mean magnetic energy density of each bubble is approximately proportional to $\epsilon_{B}$. The global energy density is then proportional to that of a single bubble multiplied by the global filling factor, so that $\bar{u}_{B} \propto \epsilon_{B} F(z) \propto \epsilon_{B} f^{\text {Pei }}$. Therefore, the global average energy density is approximately the same in the RLQ and BALQSO models: the increased number of sources in the BALQSO model compensates for the weaker magnetic fields within the bubbles of that model. Nevertheless, the local dynamics is quite different. For the RLQ model, we find that $\bar{u}_{B} / u_{\mathrm{fid}} \approx F(z)$ at $z \lesssim 7$. This indicates that, in regions filled by magnetized bubbles, the magnetic and thermal pressures have reached approximate equipartition in this model. On the other hand, the BALQSO model has $\bar{u}_{B} / u_{\mathrm{fid}} \approx 0.1 F(z)$, so that thermal energy still dominates inside and around the bubbles.

The bottom panel of Figure 5 shows the separate contributions to the ratio between the volume-normalized magnetic energy density $\bar{u}_{B} /\left[\epsilon_{-1} F(z)\right]$ and $u_{\mathrm{fid}}$ for the RLQ model by quasars at $z_{0}>4$ and at $z_{0}<4$, with the same parameter choices as in the top panel. In each case, we divide the magnetic energy density by the appropriate filling factor in order to display the magnitude of the magnetic energy in the volume filled by bubbles rather than the global volume average. The assumed quasar lifetime does not have a strong effect on the magnetic energy densities in the bubbles. The ratio increases with the quasar lifetime because longer lifetime sources perform a greater proportion of their total expansion during the active phase, so that expansion losses are less severe. As expected, the importance of the magnetic energy relative to the ambient thermal energy in the region filled with bubbles declines with redshift. For $\tau_{q}=10^{7} \mathrm{yr}$, we find that $\sim 15 \%$ of space is filled at $z=3$ with magnetic fields that are, on average, slightly below equipartition, while a much smaller fraction of space $(\sim 0.3 \%)$ contains strong fields. The remnants of highredshift quasars fill approximately $20 \%$ of space at $z=0$ if $\tau_{q}=10^{7} \mathrm{yr}$; in these regions, the average magnetic field strength corresponds to $u_{B} \sim 0.03 u_{\mathrm{fid}}$. The much younger remnants of low-redshift quasars fill $\sim 20 \%$ of space with stronger field regions (where $u_{B} \sim 0.8 u_{\mathrm{fid}}$, on average, if $\tau_{q}=10^{7} \mathrm{yr}$ ). We stress that these magnetic energy values are averages. More details on the distribution of field strengths at different epochs are given in the following section.

As mentioned in $\S 2.1 .4$, our model underestimates the ambient pressure at low redshifts because many sources are embedded within galaxy groups or clusters. The gas in such groups not only is at a higher density than the diffuse IGM but also has a higher temperature (approximately the virial temperature of the group or cluster). Figure 5 shows that even if the higher pressure in groups can suppress the expansion of bubbles around low- $z$ quasars, a high- $z$ component in the RLQ model will nevertheless pollute $\sim 20 \%$ of space with low-level fields. Of course, the enhanced pressure inside groups will not halt the formation of quasars.
Therefore, magnetic energy will still be expelled into the intragroup medium and possibly get mixed by motions of galaxies or mergers with other groups. The outcome may be that the groups collapse with a low-level field, which subsequent outflows enrich by injecting magnetic energy directly into the intragroup or intracluster medium. The net result would be little different from the picture outlined above, except that the high-field regions created by low- $z$ quasars would be strongly correlated with groups and clusters.

\subsection{Bubble Size and Magnetic Field Distribution}

Next we would like to find the probability distribution functions of the bubble magnetic field and radius. Let $P(B, z) d B$ be the probability at redshift $z$ that a random point in space is contained within a bubble having $\left\langle B^{2}\right\rangle^{1 / 2}$ in the range $(B, B+d B)$. The probability at redshift $z$ that the bubble was produced by a quasar that formed at $z^{\prime}$ is

$$
P\left(B, z, z^{\prime}\right) d B \propto \hat{V}\left(z, M_{h}, z^{\prime}\right)\left(\frac{d n_{q}}{d M_{h} d z^{\prime}}\right) \frac{d M_{h}\left(B, z, z^{\prime}\right)}{d B} d B .
$$

Then, $P(B, z) d B \propto d B \int_{z}^{\infty} P\left(B, z, z^{\prime}\right) d z^{\prime}$. As described in $\S 3.1$, the magnetic energy density inside a bubble scales linearly with $\epsilon_{B}$; therefore, we discuss $P(\widetilde{B}, z)$, which is approximately independent of $\epsilon_{B}$.

Figure 6 presents the probability distribution $P(\widetilde{B}, z)$ of magnetic fields for the RLQ model at a series of redshifts $(z=0,3,6,9,12$, and 15 , from left to right). All curves are normalized to have a total area of unity and assume $f_{R}^{\mathrm{PS}}=$ $0.01, f_{R}^{\mathrm{Pei}}=0.1, f_{d}=0.8$, and $\tau_{q}=10^{7}$ yr. The pronounced asymmetry in the distribution function at low redshifts is

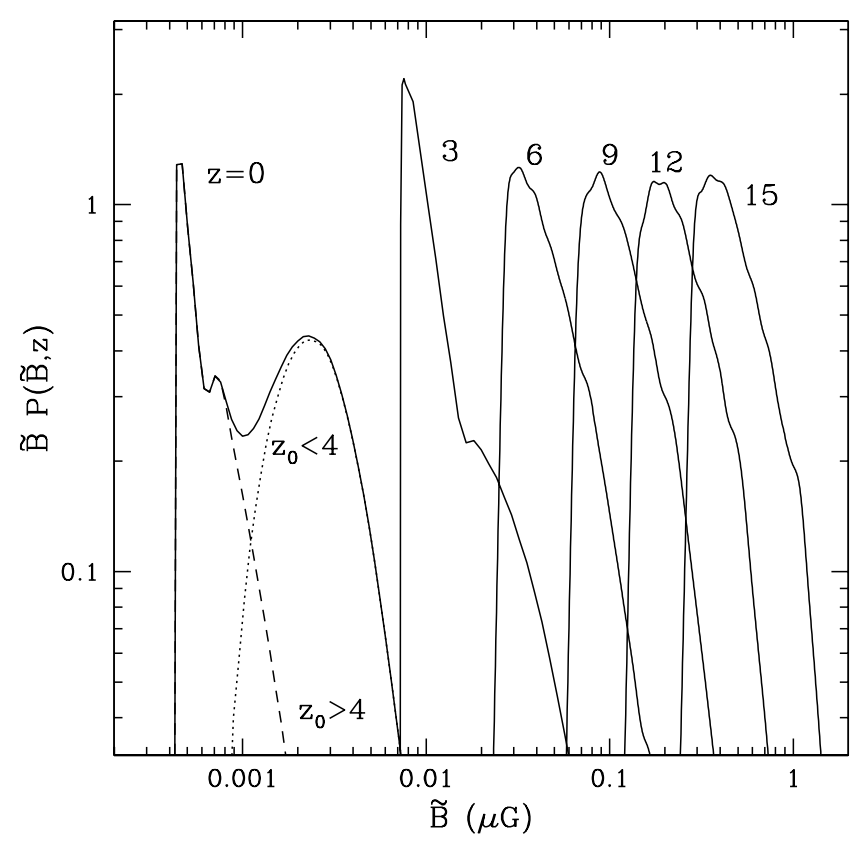

FIG. 6.-Probability distribution of bubble magnetic field, $P(\tilde{B}, z)$, for $z=0,3,6,9,12$, and 15 , from left to right, where $\tilde{B}=B /\left(\epsilon_{B} / 0.1\right)^{1 / 2}$. The dotted curve shows the contribution to the $z=0$ distribution function from quasars at $z_{0}<4$, and the dashed curve shows the contribution from quasars at $z_{0}>4$. The calculation assumes $f_{R}^{\mathrm{PS}}=0.01, f_{R}^{\mathrm{Pei}}=0.1, f_{d}=0.8$, $\tau_{q}=10^{7} \mathrm{yr}, \epsilon_{B}=0.1$, and $z_{r}=7$. 
due to the substantial increase in the minimum halo mass after reionization. The rise at high $B$ in the $z=0$ curve is because low-redshift halos tend to be large. Magnetic flux conservation and equation (22) imply that $B \propto M_{h}^{1 / 5}$, if the quasar redshift is held constant. The quasar era produces a surplus of massive halos, and the corresponding bias to large magnetic fields can be clearly seen by the dotted line in Figure 6, which shows the contribution of quasars with $z_{0}<4$ to $P(\tilde{B}, 0)$. The dashed line shows the contribution from quasars at high redshifts $\left(z_{0}>4\right)$; these make up the low-field regions.

The probability distribution of comoving bubble radii, $P(\hat{R}, z)$, is calculated in an exactly analogous fashion and is shown in Figure 7 for the same series of redshifts $(z=15,12$, $9,6,3$ and 0 , from left to right) and same parameter choices as in Figure 6. This distribution function is approximately independent of $\epsilon_{B}$ [by eq. (22), $\hat{R}_{\max } \propto\left(1+\epsilon_{B}\right)^{1 / 5}$; see Fig. $3]$. The similarity in trends between the two figures is clear. The dotted curve again shows the contribution to the $z=0$ distribution function from quasars with $z_{0}<4$, while the dashed curve shows the contribution from $z_{0}>4$ quasars. Note that the low- $z$ distribution is strongly biased toward large bubbles because the contributing halos are massive.

Because the probability distribution of bubble radii depends only weakly on $\epsilon_{B}$ while the peak of the magnetic field distribution scales approximately as $B_{\text {peak }} \propto \epsilon_{B}^{1 / 2}$, the BALQSO model produces bubbles with characteristic sizes very similar to those of the RLQ model, with each bubble containing weaker magnetic fields. A minimum mass cutoff shifts the peak to a larger radius and magnetic field strength because higher mass halos generically produce larger bubbles with stronger magnetic fields. Setting $f_{d}=0$ shifts $P(\hat{R}, z)$ to smaller radii (with mean size $\sim 1 \mathrm{Mpc}$ at $z=0$ ) but also shifts $P(B, z)$ toward stronger magnetic fields (with

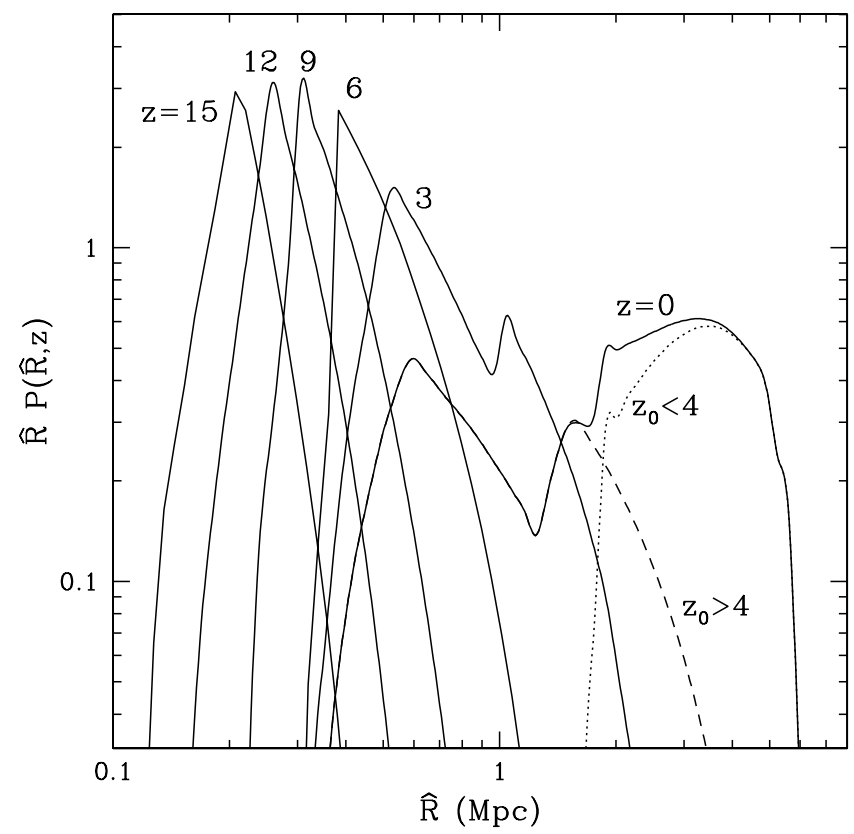

FIG. 7.-Probability distribution of bubble comoving radius $P(\hat{R}, z)$, for $z=15,12,9,6,3$, and 0 , from left to right. The dotted curve shows the contribution to the $z=0$ distribution function from quasars at $z_{0}<4$, and the dashed curve shows the contribution from quasars at $z_{0}>4$. The calculation assumes $f_{R}^{\mathrm{PS}}=0.01, f_{R}^{\mathrm{Pei}}=0.1, f_{d}=0.8, \tau_{q}=10^{7} \mathrm{yr}, \epsilon_{B}=0.1$, and $z_{r}=7$. a mean at $\left.B \sim 2 \times 10^{-9} \mathrm{G}\right)$ because a bubble with a given magnetic energy input is physically smaller, and hence its fields are less diluted.

\subsection{Reionization by Quasar Outflows}

This model, with minor modifications, also allows us to examine the feasibility of cosmological reionization by quasar outflows. We use an approach similar to that of Tegmark et al. (1993), who examined collisional reionization due to starburst winds from low-mass galaxies in the early universe.

In this case, we must track the ionization history of each bubble. A simple way to do so is to follow the temperature of the interior of each bubble (eq. [18]). As described in $\S 2.1 .3$, if $T_{b}>1.5 \times 10^{4} \mathrm{~K}$, the hot gas in the bubble will ionize a fraction $f_{m} \sim 0.1$ of the incoming hydrogen; if the bubble temperature is below this critical value, incoming hydrogen will remain neutral. An estimate of the ionized volume produced by each bubble is therefore its comoving volume when this critical temperature is reached, $\hat{V}_{T}$. Later, the ionized hydrogen may recombine on a timescale $\left(\alpha_{\mathrm{B}} n_{b}\right)^{-1}$, where $\alpha_{\mathrm{B}}$ is the case $\mathrm{B}$ recombination coefficient for hydrogen. We find that, because of the small internal density of the bubbles, very few bubbles recombine before $z \sim 5$. Recent observations of high- $z$ quasars show that reionization occurred at $z \gtrsim 6$ (Fan et al. 2000), and so we neglect the effects of recombination in the bubbles.

Given the ionized volume around each quasar, we can now find the filling factor of ionized material, $F_{r}(z)$, in an analogous manner to the filling factor of the magnetized bubbles; we need only replace $\hat{V}$ by $\hat{V}_{T}$ in equation (28). In this calculation, the minimum halo mass is determined by the condition for atomic cooling. We include quasars at $z_{0}>4$ only, so the source counts are determined by the Press-Schechter formalism through equation (26).

Note that an estimate of the ionized mass fraction would be very different than that calculated here. A fraction (1 $\left.-f_{m}\right) \sim 0.9$ of the material in the blast wave is contained in the shell, which cools much faster than the hot bubble cavity and, because of its higher density, will also recombine faster than the cavity. Therefore, our scenario will be considerably more efficient at ionizing a large volume of space than at reionizing a large fraction of the hydrogen atoms.

As shown by equation (22), $\epsilon_{B}$ has only a small effect on the final radius of the bubbles, so collisional reionization is nearly independent of the magnetic field strength. The BALQSO model is thus most efficient at reionization (because $f_{\mathrm{BAL}}=10 f_{R}$ ), and so we focus on that model in this section.

The reionization filling factor, $F_{r}(z)$, is plotted in Figure 8. For illustrative purposes, we show results for the two extreme cases, $f_{d}=1$ (solid line) and $f_{d}=0$ (short-dashed line); each of these assumes that $\tau_{q}=10^{7} \mathrm{yr}, f_{\mathrm{BAL}}^{\mathrm{PS}}=0.1$, and $\epsilon_{B}=0.01$. Also shown are cases in which $\tau_{q}=10^{6} \mathrm{yr}$, $f_{\mathrm{BAL}}^{\mathrm{PS}}=1.0$, and $\epsilon_{B}=0.01$, with $f_{d}=0$ (long-dashed line) and $f_{d}=1$ (dot-dashed line). (The last two models illustrate different ways to match the high- and low- $z$ source counts; see the discussion accompanying eq. [27].)

Clearly, only in the most optimistic scenarios is complete reionization by quasar outflows possible. Very high rates of injection of dissipated energy into the bubble interiors $\left(f_{d} \sim 1\right)$ are required in order to reionize even $\sim 80 \%$ of space before the existing observational limit $(z \sim 6)$. More conservative scenarios predict that only $\sim 50 \%$ of space can 


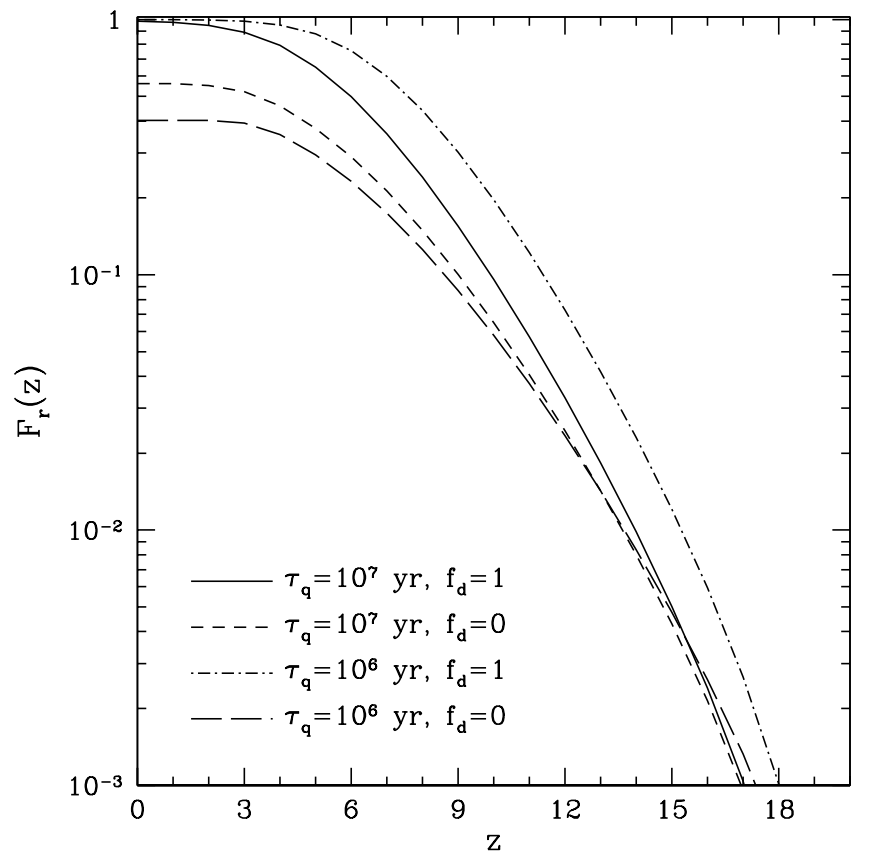

FIG. 8.-Space filling fraction of ionized material, $F_{r}(z)$, as a function of redshift, for several different scenarios. The quasar population with $z_{0}>4$ is included. Results are shown for $f_{d}=1$ (solid line) and $f_{d}=0$ (short-dashed line); each of these cases has $f_{\mathrm{BAL}}^{\mathrm{PS}}=0.1, \tau_{q}=10^{7} \mathrm{yr}$, and $\epsilon_{B}=0.01$. Also shown are cases in which $f_{\mathrm{BAL}}^{\mathrm{PS}}=1.0, \tau_{q}=10^{6} \mathrm{yr}$, and $\epsilon_{B}=0.01$, with $f_{d}=0$ (long-dashed line) and $f_{d}=1$ (dot-dashed line). The minimum halo mass is determined by the requirement of efficient atomic cooling at all redshifts.

be ionized by the quasar bubbles even at the present day. By this point, recombination inside the bubbles has further reduced the filling factor by $\sim 30 \%$. We conclude that collisional ionization by quasar outflows is a less viable scenario for the reionization of the IGM than is photoionization (see, e.g., Gnedin 2000).

\section{DISCUSSION}

We predict that the IGM has a cellular magnetic structure, with highly magnetized bubbles representing the old remnants of quasar outflows. Different types of outflows result in different outcomes in our models. RLQs at low redshifts $\left(\tau_{q}=10^{7} \mathrm{yr}, \epsilon_{B} \gtrsim 0.1, f_{R}^{\mathrm{Pei}} \gtrsim 0.1\right.$, and $\left.z_{0}<4\right)$ generate high-field regions that fill $\sim 20 \%$ of the photoionized IGM volume with regions in which the magnetic and thermal energy densities are near equipartition. An (as yet unobserved) population of high-redshift RLQs $\left(\tau_{q}=10^{7} \mathrm{yr}\right.$, $\epsilon_{B} \gtrsim 0.1, f_{R}^{\mathrm{PS}} \gtrsim 0.01$, and $z_{0}>4$ ) fills a further $\sim 20 \%$ of space with bubbles in which the magnetic energy density is $\sim 3 \%$ of the thermal energy density. However, the potentially less magnetized outflows from BAL quasars $\left(\tau_{q}=10^{7}\right.$ $\mathrm{yr}, \epsilon_{B} \gtrsim 0.01, f_{\mathrm{BAL}}^{\mathrm{Pei}} \sim 1$, and $f_{\mathrm{BAL}}^{\mathrm{PS}} \gtrsim 0.1$ ) may fill most of space with a weaker magnetic field. In reality, a combination of these processes will likely have produced a lowlevel field filling most of space, with high-field peaks generated by RLQ activity. As shown by Figure 5, these results are not sensitive to the assumed quasar lifetime.

Figure 5 also shows that sources at both high and low redshifts can have substantial effects on the IGMF; our model predicts that the contributions to the total filling factor of quasars at $z_{0}<4$ (which we describe using the observed luminosity function) and those at $z_{0}>4$ (which we describe through the Press-Schechter mass function) are comparable. However, because the high- $z$ sources reside in less massive halos and are therefore generically less luminous and because the bubbles produced by these sources have expanded for longer times, the volume filled by the high- $z$ sources has a considerably weaker magnetic field than that filled by the observed low- $z$ population. Imposing a low-mass cutoff of $M_{h, \min }=10^{11} M_{\odot}$ on RLQs hosting outflows will essentially eliminate any contribution from high- $z$ sources, but it only reduces the filling factor from low- $z$ sources to $\sim 15 \%$. Sources with $M_{h} \geq 10^{12} M_{\odot}$ fill $\sim 5 \%$ of space at the present day, so even a very extreme assumption about the requirements for quasar outflows leaves a nonnegligible filling factor for magnetized material. Note that there is no evidence of such a lower mass limit for BAL systems.

Our model provides only an upper limit to the coherence length of the IGMF because we do not follow the field structure inside each bubble. An observational limit on this coherence length can be obtained from the mean Faraday rotation signal of distant sources. To estimate an upper limit to this effect from our model, we assume that the field within each bubble is coherent over the entire bubble diameter and that all bubbles at a given redshift are identical, with the characteristic size $r(z)$ given by the mean of the probability distribution of bubble radii. The number density of bubbles at a redshift $z$ is then $N(z)=3 \phi(z) /$ $\left[4 \pi r^{3}(z)\right]$, where we use $\phi(z)$ rather than $F(z)$ in order to include the possibility of overlapping bubbles. The probability per unit path length of encountering a bubble centered at $z$ is $\pi N(z) r^{2}(z)$, so the expected squared rotation angle $d \varphi^{2}$ from bubbles in the redshift interval $(z, z+d z)$ is

$$
d \varphi^{2}=\left[\frac{4 r(z)}{3} \frac{e^{3}}{2 \pi m_{e}^{2} c^{2} v^{2}} n_{e}(z) \frac{B(z)}{\sqrt{2}}\right]^{2} \pi N(z) r^{2}(z)\left|\frac{c d t}{d z}\right| d z,
$$

where $n_{e}(z)$ is the electron number density, $m_{e}$ is the electron mass, $e$ is the electron charge, $v_{0}=v /(1+z)$ is the observed frequency, and $B(z)$ is the redshift-dependent mean bubble magnetic field strength. We have assumed a random impact parameter through each bubble and that the magnetic field of each bubble is randomly oriented with respect to the line of sight. Faraday rotation results are usually quoted in terms of the rotation measure, $R M=\Delta\left(\left\langle\varphi^{2}\right\rangle^{1 / 2}\right) / \lambda_{0}^{2}$, where $\lambda_{0}=c / v_{0}$. For our standard RLQ and BALQSO scenarios, $\mathrm{RM}(z=20) \sim 0.7 \mathrm{rad} \mathrm{m}^{-2}$ and $\operatorname{RM}(z=2.5) \sim 0.5 \mathrm{rad}$ $\mathrm{m}^{-2}$. Observations limit $\mathrm{RM}(z=2.5) \lesssim 5 \mathrm{rad} \mathrm{m}^{-2}$ (Kronberg 1994), an order of magnitude above our upper limit. Thus, the coherence length of the field could easily stretch across the entire scale of each bubble without violating existing constraints.

Interestingly, an early generation of quasars will have left magnetized remnants in the present-day IGM. It may be possible to observe these "fossil bubbles" through two techniques. The most direct method would be to observe Faraday rotation of a background source through the bubbles. Unfortunately, the rotation through a single bubble would be extremely weak, even assuming a coherent field across the entire bubble oriented along the line of sight (RM $\sim 3 \times 10^{-4} \mathrm{rad} \mathrm{m}^{-2}$ for a typical bubble at $z=0$ with $R \sim 1 \mathrm{Mpc}$ and $B \sim 10^{-9} \mathrm{G}$ ). Alternatively, it may be pos- 
sible to observe the synchrotron halos produced by these bubbles, if some of them contain a population of relativistic electrons. Such electrons could be produced in shocks in the IGM (see below) or by compression of the radio plasma during the formation of galaxy clusters (Enßlin \& GopalKrishna 2001). In fact, "diffuse radio halos" with sizes of $\sim 1 \mathrm{Mpc}$ are observed around the cores of many X-ray clusters, and "radio relics," somewhat smaller radio halos, are often observed near the periphery of clusters. The observed correlation of both radio relics and halos with merging clusters (Feretti 2000) suggests that shock waves from structure formation can accelerate electrons to relativistic energies and thus "turn on" the synchrotron halos. Similar processes would occur for our bubbles, although the low densities and magnetic field strengths in the uncompressed IGM imply that the halos have only a very low surface brightness (Waxman \& Loeb 2000).

The implied magnetic fields should lead to the acceleration of electrons and protons to relativistic energies in the strong intergalactic shocks that are produced by converging flows as large-scale structure forms in the IGM. The inverse Compton scattering of the microwave background by the accelerated electrons could account for a substantial fraction of the $\gamma$-ray background (Loeb \& Waxman 2000) and would also be accompanied by radio synchrotron emission due to the same electrons (Waxman \& Loeb 2000). Observations of the $\gamma$-ray and radio emission in the brightest shocks around clusters of galaxies can be used to calibrate the magnetic field strength in these regions and to test our model. Unlike Faraday rotation measurements, this approach is not sensitive to the coherence length of the magnetic field (since the typical Larmor radius of the electrons is negligible, $\lesssim 1 \mathrm{pc}$ ). The future GLAST mission $^{1}$ will have the sensitivity and angular resolution necessary to map the predicted $\gamma$-ray luminosity of galaxy clusters and correlate it with low-frequency radio maps of the same regions (Loeb \& Waxman 2000; Waxman \& Loeb 2000).

As the IGM gas collapses to form bound systems, such as galaxies and clusters of galaxies, the magnetic field is compressed further adiabatically with $u_{B}=B^{2} / 8 \pi \propto \rho_{g}^{4 / 3}$. The thermal energy density increases even faster in the adiabatic regime, $u_{T} \propto \rho_{g}^{5 / 3}$, and could be enhanced further as the specific entropy of the gas is increased at the virialization shock around these collapsed systems. Hence, as long as gas cooling is unimportant (e.g., outside the core of X-ray clusters), the magnetic field is expected to make only a small contribution to the total pressure. The typical values for our predicted IGMF at the mean IGM density translate to a mixed magnetic field strength of $\sim 0.15 \epsilon_{-1}^{1 / 2} \mu \mathrm{G}\left(\sim 1.5 \epsilon_{-1}^{1 / 2}\right.$ $\mu \mathrm{G}$ ) at an overdensity of $\sim 10^{3}$ in the RLQ (BALQSO) model, comparable to the inferred field amplitude on megaparsec scales around X-ray clusters (Fusco-Femiano et al. 1999; Rephaeli et al. 1999; Kaastra et al. 1999; Kim et al. 1989). The most important unknown parameter in our model is $\epsilon_{B}$, the ratio of magnetic to kinetic energy in quasar outflows. Shear flows during cluster collapse could enhance the IGMF (Dolag, Bartelmann, \& Lesch 1999) and compensate for low values of $\epsilon_{B}$.

This model for the formation of the IGMF has several advantages over other scenarios. The fields are produced and amplified in the accretion flow around a central black

\footnotetext{
${ }^{1}$ See http://www-glast.stanford.edu.
}

hole, for which the amplification time is short (Balbus \& Hawley 1991; Colgate \& Li 2000), so that fields in high- $z$ galaxies (Oren \& Wolfe 1995) may be easily accommodated. Compared to a cosmological origin in the early universe, the fields are produced sufficiently late that causality does not limit the comoving coherence length. Unlike the scenario of Kulsrud et al. (1997), there is a natural large-scale coherence length in this model, the characteristic bubble size at each redshift. However, note that we do not follow the geometry of the magnetic field, so the actual coherence length may be smaller. Closer study of the magnetic field evolution in radio cocoons and other outflows is needed in order to address this question, along with the related issues of the importance of magnetic reconnection.

Kronberg, Lesch, \& Hopp (1999) proposed that winds from starbursting dwarf galaxies in the early universe could carry the magnetic fields generated in stars into the IGM. Similarly to our model, they find that an early population of sources can easily pollute $\gtrsim 10 \%$ of space with magnetic fields. A starburst model for magnetic field generation, with a large number of sources having relatively small magnetic energy fractions, would be most similar to our BALQSO model, although individual magnetized bubbles are likely to be smaller in a starburst model. However, the coherence length of the field should be extremely small in this case as a result of the large number of stars contributing to each bubble (in contrast to the single source in our model). Kronberg et al. (1999) are forced to make recourse to amplification processes during the wind expansion in order to create a coherent field on subgalactic scales $(\sim 8 \mathrm{kpc}$, the size of the wind bubble in the nearby starburst galaxy M82) and subsequent "acausal diffusion" in order to create larger coherent fields.

Finally, we also found that mechanical outflows from quasars most likely cannot reionize the universe before the observed limit, $z_{r} \gtrsim 6$, even under the most favorable circumstances. Photoionization by stars or quasars is therefore required.

The implementation of our quasar outflow model into a numerical simulation of the IGM can be used to explore the spectroscopic fingerprints that the magnetic bubbles leave on the Ly $\alpha$ forest. Bryan et al. (1999) and Theuns et al. (1998) have found that existing simulations of the Ly $\alpha$ forest underestimate the median Doppler width of the absorption lines by $\sim 50 \%$ as compared to the observed median width, despite the close agreement in simulated and observed column density distributions. To date, numerous explanations have been offered, most postulating extra heat sources for the IGM not included in the simulations (Cen \& Bryan 2001 and references therein). In our model, the IGMF will provide a nonthermal contribution to the pressure of the gas. An equipartition field will increase the line width by a factor of $\sqrt{2}$, which would account for the claimed discrepancy. Such regions fill $\sim 20 \%$ of space at $z=3$ in our model, suggesting that nonthermal pressure from magnetic fields may play a significant role in determining these line widths.

We thank Rennan Barkana and Martin Rees for helpful comments on the manuscript. This work was supported in part by NASA grants NAG 5-7039 and 5-7768, and NSF grants AST 99-00877 and AST 00-71019 for A. L.; S. R. F. acknowledges the support of an NSF graduate fellowship. 
Balbus, S. A., \& Hawley, J. F. 1991, ApJ, 376, 214

Barkana, R., \& Loeb, A. 2001, Phys. Rep., 349, 125

Beck, R., et al. 1996, ARA\&A, 34, 155

Begelman, M. C., Blandford, R. D., \& Rees, M. J. 1984, Rev. Mod. Phys., 56,255

Bertschinger, E. 1985, ApJS, 58, 39

Bicknell, G. V., Cameron, R. A., \& Gingold, R. A. 1990, ApJ, 357, 373

Biretta, J. A., Zhou, F., \& Owen, F. N. 1995, ApJ, 447, 582

Blandford, R. D., \& Begelman, M. C. 1999, MNRAS, 303, L1

Blandford, R., \& Eichler, D. 1987, Phys. Rep., 154, 1

Bridle, A. H., \& Perley, R. A. 1984, ARA\&A, 22, 319

Bryan, G. L., Machacek, M., Anninos, P., \& Norman, M. L. 1999, ApJ, 517, 13

Bryan, G., \& Norman, M. 1998, ApJ, 495, 80

Cen, R., \& Bryan, G. L. 2001, ApJ, 546, L81

Cen, R., \& Ostriker, J. P. 1999, ApJ, 514, 1

Chiu, W. A., \& Ostriker, J. P. 2000, ApJ, 534, 507

Chokshi, A., \& Turner, E. L. 1992, MNRAS, 259, 421

Colgate, S., \& Li, H. 2000, in ASP Conf. Ser. 334, Highly Energetic Physi-

cal Processes and Mechanisms for Emission from Astrophysical Plasmas, ed. P. C. H. Martens, S. Tsuruta, \& M. Weber (San Francisco: ASP), 255

Cox, D. P. 1972, ApJ, 178, 159

Daly, R. A., \& Loeb, A. 1990, ApJ, 364, 451

Davé, R., Hernquist, L., Katz, N., \& Weinberg, D. H. 1999, ApJ, 511, 521

de Kool, M., \& Begelman, M. C. 1995, ApJ, 455, 448

Dolag, K., Bartelmann, M., \& Lesch, H. 1999, A\&A, 348, 351

Dreher, J. W., Carilli, C. L., \& Perley, R. A. 1987, ApJ, 316, 611

Efstathiou, G. 1992, MNRAS, 256, 43

Elvis, M., et al. 1994, ApJS, 95, 1

Enßlin, T. A., \& Gopal-Krishna 2001, A\&A, 366, 26

Fabbiano, G. 1989, ARA\&A, 27, 87

Falle, S. 1981, MNRAS, 195, 1011

Fan, X., et al. 2000, AJ, 120, 1167

Feretti, L. 2000, in The Universe at Low Radio Frequencies, to appear in ASP Conf. Ser. (astro-ph/0006379)

Fusco-Femiano, R., et al. 1999, ApJ, 513, L21

Gallagher, S. C., et al. 1999, ApJ, 519, 549 2001, ApJ, 546, 795

Ge, J. P., \& Owen, F. N. 1993, AJ, 105, 778

Gebhardt, K., et al. 2000, ApJ, 543, L5

Gnedin, N. Y. 2000, ApJ, 535, 530

Gnedin, N. Y., Ferrara, A., \& Zweibel, E. G. 2000, ApJ, 539, 505

Gnedin, N. Y., \& Ostriker, J. P. 1997, ApJ, 486, 581

Haiman, Z., \& Loeb, A. 1997, ApJ, 483, 21 1998, ApJ, 503, 505

Harrison, E. R. 1973, MNRAS, 165, 185

Howard, A. M., \& Kulsrud, R. M. 1997, ApJ, 483, 648

Hoyle, F. 1969, Nature, 223, 936

Ikeuchi, S., Tomisaka, K., \& Ostriker, J. P. 1983, ApJ, 265, 583

Kaastra, J. S., et al. 1999, ApJ, 519, L119

Kaiser, C. R. 2000, A\&A, 362, 447

Kim, K.-T., Kronberg, P. P., Dewdney, P. E., \& Landecker, T. L. 1990, ApJ, 355, 29

Kim, K.-T., Kronberg, P. P., Giovannini, G., \& Venturi, T. 1989, Nature, 341,720

\section{REFERENCES}

Kim, K.-T., Tribble, P. C., \& Kronberg, P. P. 1991, ApJ, 379, 80

Koyama, K., et al. 1995, Nature, 378, 255

Krolik, J. H. 1999, Active Galactic Nuclei (Princeton: Princeton Univ. Press)

Kronberg, P. P. 1994, Rep. Prog. Phys., 57, 325

Kronberg, P. P., Lesch, H., \& Hopp, U. 1999, ApJ, 511, 56

Kulsrud, R. M., Cen, R., Ostriker, J. P., \& Ryu, D. 1997, ApJ, 480, 481

Laing, R. A. 1980, MNRAS, 193, 439

Laor, A. 2000, ApJ, 543, L111

Loeb, A., \& Waxman, E. 2000, Nature, 405, 156

Magorrian, J., et al. 1998, AJ, 115, 2285

Martel, H., \& Shapiro, P. R. 2001, in Rev. Mexicana Astron. Astrofis., Ser. de Conf. 10, The Seventh Texas-Mexico Conference on Astrophysics: Flows, Blows, and Glows, ed. W. H. Lee \& S. Torres-Peimbert (Mexico, DF: Inst. Astron, UNAM), 101

Medina-Tanco, G., \& Enßlin, T. A. 2000, Astropart. Phys., in press (astro$\mathrm{ph} / 0011454)$

Murray, N., Chiang, J., Grossman, S. A., \& Voit, G. M. 1995, ApJ, 451, 498

Natarajan, P., \& Sigurdsson, S. 1999, MNRAS, 302, 288

Natarajan, P., Sigurdsson, S., \& Silk, J. 1998, MNRAS, 298, 577

Navarro, J., Frenk, C., \& White, S. 1997, ApJ, 490, 493 (NFW)

Navarro, J., \& Steinmetz, M. 1997, ApJ, 478, 13

Oren, A. L., \& Wolfe, A. M. 1995, ApJ, 445, 624

Ostriker, J. P., \& McKee, C. F. 1988, Rev. Mod. Phys., 60, 1

Pei, Y. 1995, ApJ, 438, 623

Perley, R. A., \& Taylor, G. 1991, AJ, 101, 1623

Press, W. H., \& Schechter, P. 1974, ApJ, 187, 425

Quashnock, J., Loeb, A., \& Spergel, D. N. 1989, ApJ, 344, L49

Rees, M. J., \& Setti, G. 1968, Nature, 219, 127

Rephaeli, Y., Gruber, D., \& Blanco, P. 1999, ApJ, 511, L21

Salucci, P., et al. 1999, MNRAS, 307, 637

Schneider, D. P., et al. 2001, AJ, 121, 1232

Stebbins, A., \& Silk, J. 1986, ApJ, 300, 1

Stern, D., et al. 2000, AJ, 119, 1526

Storrie-Lombardi, L. J., et al. 2001, MNRAS, 322, 933

Sutherland, R. S., \& Dopita, M. A. 1993, ApJS, 88, 253

Tanimori, T., et al. 1998, ApJ, 497, L25

Taylor, G. B., et al. 1990, ApJ, 360, 41

Taylor, G. B., \& Perley, R. A. 1993, ApJ, 416, 554

Tegmark, M., Silk, J., \& Evrard, A. 1993, ApJ, 417, 54

Theuns, T., Leonard, A., Efstathiou, G., Pearce, F. R., \& Thomas, P. R. 1998, MNRAS, 301, 478

Thoul, A. A., \& Weinberg, D. H. 1996, ApJ, 465, 608

Tingay, S. J., et al. 2001, ApJ, 549, 55

Turnshek, D. A., et al. 1994, ApJ, 428, 93

Valageas, P., \& Silk, J. 1999, A\&A, 347, 1

Voit, G. M. 1996, ApJ, 465, 548

Waxman, E., \& Loeb, A. 2000, ApJ, 545, L11

Weymann, R. J. 1997, in ASP Conf. Ser. 128, Mass Ejection from AGN, ed. N. Arav, I. Shlosman, \& R. J. Weymann (San Francisco: ASP), 3

Willott, C. J., Rawlings, S., Blundell, K. M., \& Lacy, M. 1999, MNRAS, 309, 1017

Zeldovich, Ya. B., Ruzmaikin, A. A., \& Sokoloff, D. D. 1983, Magnetic Fields in Astrophysics (New York: Gordon and Breach) 